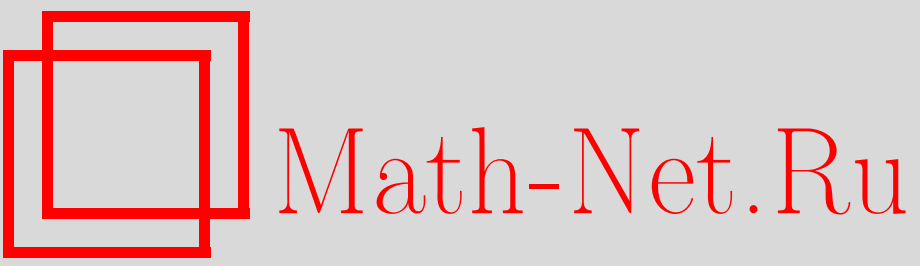

М. М. Глухов, О рассеивающих линейных преобразованиях для блочных шифрсистем, Матем. вопр. криптогр., 2011, том 2, выпуск 2, 5-39

DOI: https://doi.org/10.4213/mvk29

Использование Общероссийского математического портала Math-Net.Ru подразумевает, что вы прочитали и согласны с пользовательским соглашением

http://www.mathnet.ru/rus/agreement

Параметры загрузки:

IP: 34.229 .45 .116

26 апреля 2023 г., 07:13:22 


\title{
О рассеивающих линейных преобразованиях для блочных шифрсистем
}

\author{
М. М. Глухов \\ Академия криптографии Российской Федерации, Москва
}

Получено 22.IV.2010

Рассматриваются блочные шифрсистемы типа AES над конечным полем с линейными рассеивающими преобразованиями трех типов: блочноравномерными, максимально рассеивающими и составными. Исследуются матрицы вероятностей переходов пар различных входных блоков в пары выходных блоков при случайном, независимом и равновероятном выборе ключей каждого раунда. Для схем без рассеивающих преобразований в нечетных раундах найдено минимальное число раундов, достаточное для порождения 2-транзитивного множества подстановок.

Ключевые слова: блочные шифрсистемы, матрица переходных вероятностей пар блоков, рассеивающее преобразование, максимально рассеивающее преобразование, блочно-равномерное преобразование

\section{On mixing linear transforms for block ciphers}

\section{M. Gluhov}

Academy of Cryptography of Russian Federation, Moscow

\begin{abstract}
We consider AES-type block ciphers over the finite field with linear mixing transforms of three classes: maximally mixing, block-uniform and composite. Transition probability matrices of pairs of input blocks for these schemes with random independent equiprobable round keys are investigated. A minimum number of rounds sufficient for the generation of doubly transitive set of permutations are found for schemes without mixing transforms in odd rounds.
\end{abstract}

Key words: block ciphers, transition probability matrix of pairs of blocks, mixing transforms, maximally mixing transform, block uniform transform

Citation: Mathematical Aspects of Cryptography, 2011, vol. 2, no. 2, pp. 5-40 (Russian). 


\section{§ 1. Введение}

Рассмотрим блочную многораундовую криптосхему типа AES над полем $G F(q)$ (см. [6]), называемую также XSL-схемой и XSL-сетью. Будем считать, что ее входными и выходными блоками являются векторы-строки длины $n$, составленные из элементов поля $G F(q)$. Множество $V_{n}(q)$ всех таких векторов с операциями покомпонентного сложения и умножения на элементы поля $G F(q)$ является линейным пространством размерности $n$ над полем $G F(q)$. Каждый блок $a$ является конкатенацией $r$ подблоков длины $m$ и записывается в виде $a=\left(a_{1}, \ldots, a_{r}\right)$, где $m r=n, m>1, r>1$. При этом вектор $a_{j} \in V_{m}(q)$ есть $j$-й подблок блока $a$. Аналогичное представление будет использоваться и для раундовых ключей. Основные операции и нулевой элемент в поле $G F(q)$ и в линейных пространствах над ним будем обозначать теми же знаками, что и во множестве целых чисел. Из контекста всегда будет видно, о чем идет речь.

В $i$-м раунде процесса шифрования блок $a$ подвергается преобразованию $F_{x}$, где

$$
F_{x}(a)=L_{i}\left(\Pi\left(\sigma_{x}(a)\right)\right), \text { или, иначе, } a F_{x}=a \sigma_{x} \Pi L_{i},
$$

$x$ - раундовый ключ, $\sigma_{x}(a)=a \sigma_{x}=a+x, \Pi-$ преобразование множества $V_{n}(q)$, осуществляемое с помощью системы из $r$ подстановок $\prod_{i}, i=1, \ldots, r$, множества $V_{m}(q)$ по правилу:

$$
\Pi(a)=\left(\Pi_{1}\left(a_{1}\right), \ldots, \Pi_{r}\left(a_{r}\right)\right),
$$

$L_{i}$ - линейное преобразование пространства $V_{n}(q)$. Множество всех преобразований, осуществляемых в $i$-м раунде шифрования, можно записать в виде $\Sigma_{n} \Pi L_{i}$, где $\Sigma_{n}=\left\{\sigma_{x}: x \in V_{n}(q)\right\}$. Для упрощения записей будем считать, что подстановка П является постоянной для всех раундов. Далее будем предполагать, что ключ $x$ выбирается случайно, равновероятно и независимо в каждом раунде.

Обозначим через $V\left(i_{1}, \ldots, i_{k}\right)$ при $1 \leq i_{1}<\ldots<i_{k} \leq r$ множество всех блоков, в которых подблоки с номерами $i_{1}, \ldots, i_{k}$ - ненулевые, а остальные подблоки - нулевые. Множество $V_{n}(q) \backslash\{0\}$ разбивается на $2^{r}-1$ попарно не пересекающихся подмножеств

$$
V(1), V(2), \ldots, V(r), V(1,2), V(1,3), \ldots, V\left(i_{1}, \ldots, i_{k}\right), \ldots, V(1,2, \ldots, r) .
$$

Очевидно, что $i$-й подблок вектора $a \sigma_{x} \Pi$ при любых $a, x \in V_{n}(q)$ зависит лишь от $i$-х подблоков векторов $a, x$. Для преодоления этого недостатка 
схемы к векторам вида $a \sigma_{x} \Pi$ применяется линейное преобразование $L_{i}$, которое выбирается так, чтобы результаты его действия на подблоки вектора зависели от многих подблоков исходного вектора. В связи с этим такие преобразования называют рассеивающими. Они обычно выбираются линейными с тем, чтобы допускали сравнительно простую реализацию и не портили нужных параметров нелинейных преобразований $\Pi_{i}$. Однако свойства рассеивающих преобразований оказывают существенное влияние не только на сложность реализации процессов зашифрования и расшифрования, но и на криптографическую стойкость схемы. В связи с этим актуальной является задача поиска сравнительно просто реализуемых рассеивающих линейных преобразований и исследования их свойств. При этом под основным рассеивающим свойством преобразования $L$ мы здесь понимаем его способность обеспечивать сравнительно быструю сходимость последовательности степеней $\left(P^{(2)}\right)^{t}$ матрицы $P^{(2)}$ переходных вероятностей пар различных блоков под действием системы подстановок $\Sigma_{n} \Pi L$ к равномерной матрице при подходящем выборе подстановок $\Pi_{i}$ и при $t \rightarrow \infty$. Элементами матрицы $P^{(2)}$ являются отношения числа подстановок системы $\Sigma_{n} \Pi L$, переводящих заданную пару блоков в заданную пару блоков, к общему числу подстановок в системе; равномерной мы называем дважды стохастическую матрицу, все элементы которой равны между собой. Ниже сходимость последовательности степеней матрицы $P^{(2)}$ к равномерной матрице мы для краткости будем называть о сходимостью матрицы $P^{(2)}$ к равномерной матрице.

В данной работе для указанной криптосхемы исследуются три класса рассеивающих линейных преобразований, называемых соответственно блочно-равномерными, максимально рассеивающими и составными.

Блочно-равномерным мы называем обратимое линейное преобразование $L$ пространства $V_{n}(q)$, удовлетворяющее условию:

$$
\begin{gathered}
|L(V(i)) \cap V(j)|=l \text { при некотором } \\
\text { фиксированном } l>0 \text { и всех } i, j=\{1, \ldots, r\} .
\end{gathered}
$$

Максимально рассеивающим называют обратимое линейное преобразование $L$ пространства $V_{n}(q)$, удовлетворяющее условию

$$
\forall a \in V_{n}(q):|w(a L)+w(a)| \geq r+1,
$$

где $w(a)$ - число ненулевых подблоков в блоке $a \in V_{n}(q)$. Заметим, что такие преобразования обладают максимальным коэффициентом рассеивания, введенным Ф. М. Малышевым как $\min |w(a L)+w(a)|$ по всем $a \in V_{q}(n)$. 
Составным при $r=d h, d>1, h>1$, мы называем линейное преобразование пространства $V_{n}(q)$, представимое в виде произведения $L=g f$, где $g$ - перестановка подблоков в блоке, а $f$ реализуется в виде системы обратимых линейных преобразований $f_{i}, i=1, \ldots, h$, причем $f_{i}$ действует на подпространстве $V_{m d}^{(i)}$, состоящем из конкатенаций подблоков с номерами $(i-1) d+1,(i-1) d+2, \ldots, i d$. Каждый вектор из $V_{m d}^{(i)}$ условимся называть $i$-м каскадом соответствующего блока. К составным относится, в частности, рассеивающее линейное преобразование в реальной криптосхеме AES. В ней блок записывается в виде матрицы над полем $G F\left(2^{8}\right)$, роль подстановки $g$ играет преобразование сдвигов строк в матрице-блоке, а роль $f_{i}$ - одно и то же максимально рассеивающее преобразование, заключающееся в умножении на подходящую матрицу над полем $G F\left(2^{8}\right)$ всех столбцов матрицы-блока, которые и являются каскадами этого блока в указанном выше смысле.

Криптосхему, в которой все линейные преобразования $L_{i}$ содержатся в любом одном из указанных трех классов, будем ради краткости называть A-схемой. Криптографическая стойкость $k$-раундовой А-схемы существенно зависит от свойств матрицы $P^{(2, k)}$ переходных вероятностей пар различных входных блоков в пары выходных блоков за $k$ раундов. В частности, близость матрицы $P^{(2, k)}$ к равномерной матрице способствует лучшему противостоянию А-схемы методам анализа, использующим разности открытых текстов. В связи с этим параметры А-схемы и число раундов ее работы при зашифровании блоков выбирают так, чтобы матрица $P^{(2, k)}$ была близка к равномерной матрице.

В общем случае вопрос о максимальном отклонении элементов матрицы $P^{(2, k)}$ от элементов равномерной матрицы является весьма сложным как с теоретической, так и с вычислительной точки зрения (в силу больших размеров матрицы $\left.P^{(2, k)}\right)$. Однако в некоторых случаях качественная оценка близости матрицы $P^{(2, k)}$ к равномерной может быть получена путем оперирования с матрицами меньших размеров. В частности, для максимально рассеивающих и блочно равномерных преобразований при подходящих П такая ситуация имеет место в криптосхемах, полученных из А-схем заменой преобразований $L_{i}$ при нечетных $i$ тождественным преобразованием. Полученные таким образом криптосхемы будем называть $\mathrm{A}_{1}$-схемами. Их основное преимущество перед А-схемами заключается в том, что в $\mathrm{A}_{1}$-схемах легче проследить изменения матриц переходных вероятностей биграмм при 
их перемножении и, в частности, при определенных ограничениях на подстановки П легко найти минимальное значение $k$ числа раундов, при котором матрица $P^{(2, k)}$ не содержит нулевых элементов, т. е. реализуется 2-транзитивное множество подстановок. Кроме того, $\mathrm{A}_{1}$-схемы имеют определенное преимущество и с точки зрения скорости шифрования и расшифрования.

Из полученных ниже результатов следует, что для $\mathrm{A}_{1}$-схем блочноравномерные рассеивающие преобразования являются оптимальными с точки зрения минимизации числа раундов для реализации 2-транзитивного множества подстановок. Однако с точки зрения скорости сходимости последовательности матриц $P^{(2, k)}$ к равномерной матрице лучшими являются максимально рассеивающие преобразования. При этом преимущество последних имеет довольно простое объяснение (см. § 5).

\section{§ 1. Общие свойства матриц переходных вероятностей пар блоков в А-схемах}

Множество преобразований блока в $i$-м раунде А-схемы при всевозможных раундовых ключах можно представить в виде $\Sigma_{n} \Pi L_{i}$. Тогда за $k$ раундов будет реализоваться система подстановок

$$
G_{k}=\left\{\sigma_{x_{1}} \Pi L_{1} \sigma_{x_{2}} \Pi L_{2} \ldots \sigma_{x_{k}} \Pi L_{k}: x_{1}, x_{2}, \ldots, x_{k} \in V_{n}(q)\right\},
$$

и матрица переходных вероятностей пар различных блоков такого шифра будет равна $P^{(2, k)}$. Заметим, что в системе (3) разные наборы $x_{1}, x_{2}, \ldots, x_{k}$ могут порождать одну и ту же подстановку, и всегда при вычислении вероятностей переходов пар блоков каждая подстановка учитывается столько раз, сколько раз она встречается в системе (3).

Так как $\Sigma_{n}$ - группа, то имеют место равенства множеств $\Sigma_{n}=\Sigma_{n} \cdot \Sigma_{n}$ и

$$
\begin{gathered}
\left(\Sigma_{n} \Pi L_{1} \Sigma_{n}\right)\left(\Sigma_{n} \Pi L_{2} \Sigma_{n}\right) \ldots\left(\Sigma_{n} \Pi L_{k-1} \Sigma_{n}\right)\left(\Sigma_{n} \Pi L_{k}\right)= \\
=\left\{\sigma_{x_{1}} \Pi L_{1} \sigma_{x_{1}^{\prime}} \sigma_{x_{2}} \Pi L_{2} \ldots \sigma_{x_{k-1}^{\prime}} \sigma_{x_{k}} \Pi L_{k}: x_{1}, x_{1}^{\prime}, x_{2}, \ldots, x_{k-1}^{\prime}, x_{k} \in V_{k}(q)\right\} .
\end{gathered}
$$

Очевидно, что система (4) будет состоять из системы (3), повторенной $q^{n(k-2)}$ раз, и потому матрицы переходных вероятностей биграмм при действии систем (3) и (4) совпадают. Ради большей симметрии мы вместо системы подстановок (4) будем рассматривать систему

$$
\left(\Sigma_{n} \Pi L_{1} \Sigma_{n}\right)\left(\Sigma_{n} \Pi L_{2} \Sigma_{n}\right) \ldots\left(\Sigma_{n} \Pi L_{k-1} \Sigma_{n}\right)\left(\Sigma_{n} \Pi L_{k} \Sigma_{n}\right) .
$$


Соответствующая этой системе матрица переходных вероятностей пар различных блоков лишь перестановкой столбцов отличается от матрицы $P^{(2, k+1)}$. Обозначим ее через $P_{1}^{(2, k)}$.

Из (4) видно, что для изучения матрицы $P_{1}^{(2, k)}$ целесообразно изучить сначала матрицу $P_{i}^{(2)}=P_{1}^{(2,1)}$ переходных вероятностей пар различных блоков при действии системы подстановок $\Sigma_{n} \Pi L_{i} \Sigma_{n}$ при фиксированном $i$. Как было замечено Б. А. Егоровым и автором (1968 г.), вероятность перехода пары блоков $(a, b)$ в пару $(c, d)$ при действии подстановок из множества $H_{1} g H_{2}$, где $H_{1}, H_{2}$ - регулярные подгруппы симметрической группы подстановок $S\left(V_{n}\right)$ (см. [7] или [2], стр. 273) и $g \in S\left(V_{n}\right)$, зависит лишь от числа подстановок системы $H_{1} g H_{2}$, отображающих разность $a-b$ в разность $c-d$. Отсюда следует, что при равновероятном и независимом выборе преобразований из системы $\Sigma_{n} \Pi L_{i} \Sigma_{n}$ матрица $P_{i}^{(2)}$ при определенной и одинаковой нумерации строк и столбцов представляется в виде тензорного произведения матриц

$$
P_{i}^{(2)}=Q^{\left(\Pi L_{i}\right)} \otimes R,
$$

где $Q^{\left(п L_{i}\right)}$ - матрица переходных вероятностей ненулевых разностей входных блоков в выходные под действием подстановок системы $\Sigma_{n} \Pi L_{i} \Sigma_{n}$, a $R$ - равномерная матрица. Отсюда и из свойств тензорного произведения следует, что

$$
P_{1}^{(2, k)}=\prod_{i=1}^{k} P_{i}^{(2)}=\left(\prod_{i=1}^{k} Q^{\left(\Pi L_{i}\right)}\right) \otimes R .
$$

Так как $R$ не влияет на основные криптографические параметры матрицы $P_{1}^{(2, k)}$, то обычно вместо матрицы $P_{1}^{(2, k)}$ изучают матрицу $Q^{(2, k)}=\prod_{i=1}^{k} Q^{\left(п L_{i}\right)}$. В частности, система подстановок (5) является 2-транзитивной тогда и только тогда, когда $Q^{(2, k)}$ не содержит нулевых элементов. В этом случае будем писать $Q^{(2, k)}>0$ и называть матрицу $Q^{(2, k)}$ положительной. Заметим еще, что действие подстановок из группы $\Sigma_{n}$ на блоки не изменяет разности блоков. Поэтому матрица переходных вероятностей ненулевых разностей под действием системы подстановок (3) совпадает с соответствующей матрицей для системы подстановок (5).

Рассмотрим $i$-й сомножитель из разложения матрицы $Q^{(2, k)}$. При этом в его записи будем опускать индекс $i$, т. е. будем рассматривать матрицу $Q^{(П L)}=\left(q_{a, b}\right), a, b \in V_{n}(q)$ при действии подстановок системы $\Sigma_{n} \Pi L \Sigma_{n}$. При тождественном $L$ матрицу $Q^{(П L)}$ будем обозначать через $Q^{(П)}$. 
Известно (см., например, [1]) что элемент $q_{a, b}$ матрицы $Q^{(\Pi L)}$ равен числу решений уравнения

$$
((a+x) \Pi) L-(x \Pi) L=b,
$$

деленному на $q^{n}$. Так как $L-$ линейное преобразование, то последнее уравнение равносильно уравнению

$$
(a+x) \Pi-x \Pi=b L^{-1} .
$$

Это означает, что матрица $Q^{(П L)}$ получается из матрицы $Q^{(П)}$ лишь перестановкой номеров ее столбцов. Если вместо номеров столбцов переставлять сами столбцы, сохраняя их нумерацию, то столбец с номером $b$ встанет на место с номером $b L$, и значит,

$$
Q^{(\Pi L)}=Q^{(\Pi)} \tilde{L},
$$

где $\tilde{L}-$ подстановочная матрица, соответствующая преобразованию $L$. Следовательно, матрица $Q^{(2, k)}$ может быть представлена в виде

$$
Q^{(2, k)}=Q^{(\Pi)} \tilde{L}_{1} Q^{(\Pi)} \tilde{L}_{2} Q^{(\Pi)} \ldots Q^{(\Pi)} \tilde{L}_{k} .
$$

Рассмотрим строение матрицы $Q^{(I)}$. Так как подстановки $\sigma_{x}$ не изменяют разностей блоков, а подстановки $\Pi_{i}$ действуют независимо на множествах $V(i)$ при $i=1, \ldots, r$, то матрицу $Q^{(I)}$ при определенном расположении ее строк и столбцов можно представить в виде клеточно-диагональной матрицы. Занумеруем ее строки и столбцы элементами из $V_{n}(q) \backslash\{0\}$ одинаково и так, чтобы векторы-номера шли последовательно из подмножеств (1). Тогда получим:

$$
Q^{(\Pi)}=\operatorname{Diag}\left(Q_{1}, Q_{2}, \ldots, Q_{r}, Q_{1,2}, Q_{1,3}, \ldots, Q_{1,2, \ldots, r}\right),
$$

где $Q_{i_{1}, \ldots, i_{k}}$ - матрица переходных вероятностей разностей из $V\left(i_{1}, \ldots, i_{k}\right)$, которая, в свою очередь, представляется в виде тензорного произведения

$$
Q_{i_{1}, \ldots, i_{k}}=Q_{i_{1}} \otimes \ldots \otimes Q_{i_{k}} .
$$

Для выражения элементов матрицы $Q_{i_{1}, \ldots, i_{k}}$ через элементы матриц $Q_{i_{1}}, \ldots, Q_{i_{k}}$ введем $\quad$ обозначения: $\quad Q_{i_{1}, \ldots, i_{k}}=\left(q_{a, b}^{\left(i_{1}, \ldots, i_{k}\right)}\right), \quad$ где $a=\left(a_{1}, \ldots, a_{r}\right)$, $b=\left(b_{1}, \ldots, b_{r}\right) \in V\left(i_{1}, \ldots, i_{k}\right)$. Тогда

$$
q_{a, b}^{\left(i_{1}, \ldots, i_{k}\right)}=q_{a_{i_{1}}, b_{i_{1}}}^{\left(i_{1}\right)} \ldots q_{a_{i_{k}}, b_{i_{k}}}^{\left(i_{k}\right)} .
$$

Нетрудно усмотреть, что элементы матрицы $Q^{(\text {П) }} \tilde{L} Q^{(\text {П) }}$ и скорость сходимости её степеней к равновероятной матрице зависят от чисел, показы- 
вающих, сколько векторов переводит $L$ из любого одного множества системы (1) в любое другое, т. е. от чисел

$$
\left|L\left(V\left(i_{1}, \ldots, i_{s}\right)\right) \cap V\left(j_{1}, \ldots, j_{t}\right)\right| .
$$

Однако точные значения элементов матрицы $Q^{(\text {П) }} \tilde{L}$, а значит, и $Q^{(П)} \tilde{L} Q^{(П)}$, зависят не только от $L$, но и от подстановки П. Эта зависимость частично исключается в $\mathrm{A}_{1}$-схемах при подходящих подстановках $\Pi_{i}$. Выберем подстановки $\Pi_{i}$ так, чтобы квадраты матриц $Q_{1}, Q_{2}, \ldots, Q_{r}$ были положительными. Тогда из свойств тензорного произведения следует, что и все диагональные клетки матрицы $\left(Q^{(П)}\right)^{2}$ будут также положительными. Заметим, что в силу теоремы, доказанной А. С. Амбросимовым (1980), при достаточно больших порядках подстановок $\Pi_{i}$ почти все они обладают указанным свойством. В общем случае подстановки с таким свойством существуют, если $q^{m}>4$; примером является подстановка обращения ненулевых элементов поля $G F(q)$. Далее будем считать, что условие $q^{m}>4$ выполнено.

Ниже при изучении $\mathrm{A}_{1}$-схем нам часто придётся умножать некоторые матрицы на матрицу $\left(Q^{(П)}\right)^{2}$ с положительными диагональными клетками. При этом будет использоваться следующее утверждение, получающееся непосредственно из правила умножения матриц.

Утверждение 1. Пусть подстановки $\Pi_{i}$ выбраны так, что квадраты клеток $Q_{1}, Q_{2}, \ldots, Q_{r}$ матрицы $Q^{(П)}$ положительны и $U-$ целочисленная матрица, строки и столбцы которой занумерованы точно так же, как и у матрицы $Q^{(\Pi)}$.

Тогда в строке с номером из $V\left(i_{1}, \ldots, i_{s}\right)$ матрищы $U\left(Q^{(\Pi)}\right)^{2}$ либо все элементы столбцов с номерами из $V\left(j_{1}, \ldots, j_{t}\right)$ ненулевые, либо все они равны 0.

Далее свойства матриц переходных вероятностей рассеивающих преобразований будут рассматриваться отдельно для каждого из указанных выше трех классов.

\section{§ 2. Блочно-равномерные рассеивающие преобразования}

Так как каждое из множеств $V(i)$ и $L(V(i)), i=1, \ldots, r$, вместе с нулем образует линейное подпространство в $V_{n}(q)$, то в условии (2), определяющем блочно равномерное преобразование, число $l$ должно иметь вид $l=q^{d}-1$ при некотором $d>0$ и $m \geq r d$. В общем случае имеет место следующее утверждение. 
Утверждение 2. Если $L-$ блочно-равномерное линейное преобразование, то $L\left(V\left(i_{1}, \ldots, i_{s}\right)\right) \cap V\left(j_{1}, \ldots, j_{t}\right) \neq \varnothing$ для любых множеств $V\left(i_{1}, \ldots, i_{s}\right)$, $V\left(j_{1}, \ldots, j_{t}\right)$ из $(1)$.

Доказательство. Из доказательства будет ясно, что, не теряя общности, можно ограничиться рассмотрением лишь множеств вида $V(1, \ldots, s)$, $V(1, \ldots, t)$. Возможны два случая: $s \geq t$ и $s<t$.

Пусть $s \geq t$. Тогда выберем векторы $\alpha_{i} \in V(i), i=1, \ldots, s$, так, что $L\left(\alpha_{i}\right) \in V(i)$ при $i=1, \ldots, t-1$ и $L\left(\alpha_{i}\right) \in V(t)$ при $i=t, \ldots, s$. Из определения $L$ следует, что такой выбор возможен. Так как система выбранных векторов линейно независима, то линейно независима и система их образов. Следовательно, векторы $\alpha=\alpha_{1}+\ldots+\alpha_{s}$ и $L(\alpha)=L\left(\alpha_{1}\right)+\ldots+L\left(\alpha_{s}\right)-$ ненулевые. Остается заметить, что $\alpha \in V(1, \ldots, s), \quad a L(\alpha) \in V(1, \ldots, t), \quad$ и потому $L(\alpha) \in L(V(1, \ldots, s)) \cap V(1, \ldots, t)$.

В случае $s<t$ выберем $\alpha_{i} \in V(i), i=1, \ldots, s$, так, что $L\left(\alpha_{i}\right) \in V(i)$, и $\alpha_{s+1}, \ldots, \alpha_{t} \in V(s)$ так, что $L\left(\alpha_{j}\right) \in V(j), j=s+1, \ldots, t$. Далее, как и выше, доказывается, что вектор $L\left(\alpha_{1}+\ldots+\alpha_{t}\right)$ содержится в $L(V(1, \ldots, s)) \cap V(1, \ldots, t)$. В итоге утверждение доказано.

Следствие 1. Если в $A_{1}$-схеме $\left(Q_{i}\right)^{2}>0$ при $i \in\{1, \ldots, r\}$, а рассеивающие преобразования в четных раундах являются блочно-равномерными, то минимальное число раундов, за которое реализуется 2-транзитивное множество подстановок, не превосходит 5. При этом на 5-м раунде достаточно лишь прибавление ключа.

Действительно, из условия $\left(Q_{i}\right)^{2}>0$ и утверждений 1,2 следует соотношение $\left(Q^{(П)}\right)^{2} L_{2}\left(Q^{(П)}\right)^{2}>0$, которое равносильно 2-транзитивности множества подстановок $\Sigma_{n} \Pi \Sigma_{n} \Pi L_{2} \Sigma_{n} \Pi \Sigma_{n} \Pi L_{4} \Sigma_{n}$, полученного за 4 раунда с последующим прибавлением ключа на 5-м раунде.

Заметим, что в общем случае четырех раундов работы $\mathrm{A}_{1}$-схемы не достаточно для реализации 2-транзитивного множества подстановок. Так, например, если $q=2, m=r d, d=1$, то матрица $\left(Q^{(\Pi)}\right)^{2} L Q^{(П)}$ при любой подстановке П не является положительной, поскольку в матрице $Q^{(П)}$ есть нулевые элементы (см. [1]).

Заметим еще, что в условиях утверждения 2 при $m>r d$ о точном значении величин (6) говорить не приходится, поскольку не известно, в какие из множеств (1) отображаются $m-r d$ базисных векторов каждого множества $V(i), i=1, \ldots, r$. Если же $m=r d$, то для величин (6) можно указать точные значения. 
Рассмотрим сначала простейший случай, когда $m=r d$ и $d=1$, т. е. $m=r, n=m^{2}$.

Выберем стандартный базис пространства $V_{n}(q)$

$$
E=\left\{e_{11}, \ldots, e_{1 m}, e_{21}, \ldots, e_{2 m}, \ldots, e_{m 1}, \ldots, e_{m m}\right\},
$$

где $e_{i j}$ - вектор из $V_{n}(q)$, у которого $j$-я координата $i$-го подблока равна 1 , а остальные координаты - нулевые. Тогда каждый вектор из $V_{n}(q)$ можно записать в виде

$$
\alpha=\left(a_{11} e_{11}+\ldots+a_{1 m} e_{1 m}\right)+\left(a_{21} e_{21}+\ldots+a_{2 m} e_{2 m}\right)+\ldots+\left(a_{m 1} e_{m 1}+\ldots+a_{m m} e_{m m}\right)
$$

с коэффициентами $a_{i j} \in G F(q)$.

Сумма слагаемых в $i$-й паре скобок этой суммы представляет собой блок, в котором все подблоки, кроме $i$-го, нулевые, а $i$-й подблок в зависимости от значений коэффициентов $a_{i j}$ может принимать любые значения из $V_{m}(q)$.

Определим линейное преобразование $L: V_{n}(q) \rightarrow V_{n}(q)$, задав его на базисных векторах следующим правилом:

$$
L\left(e_{i j}\right)=c_{j 1 i} e_{j 1}+c_{j 2 i} e_{j 2}+\ldots+c_{j m i} e_{j m}, i, j \in\{1, \ldots, m\},
$$

где коэффициенты $c_{j k i}$ выбраны с единственным условием: преобразование $L$ должно быть обратимым. Матрица $C$ этого линейного преобразования в базисе $E$ имеет следующий вид:

$$
C=\left(\begin{array}{ccccccccccccc}
c_{111} & 0 & \ldots & 0 & c_{112} & 0 & \ldots & 0 & \ldots & c_{11 m} & 0 & \ldots & 0 \\
c_{121} & 0 & \ldots & 0 & c_{122} & 0 & \ldots & 0 & \ldots & c_{12 m} & 0 & \ldots & 0 \\
\ldots & \ldots & \ldots & \ldots & \ldots & \ldots & \ldots & \ldots & \ldots & \ldots & \ldots & \ldots & \ldots \\
c_{1 m 1} & 0 & \ldots & 0 & c_{1 m 1} & 0 & \ldots & 0 & \ldots & c_{1 m m} & 0 & \ldots & 0 \\
0 & c_{211} & \ldots & 0 & 0 & c_{212} & \ldots & 0 & \ldots & 0 & c_{21 m} & \ldots & 0 \\
0 & c_{221} & \ldots & 0 & 0 & c_{222} & \ldots & 0 & \ldots & \ldots & c_{22 m} & \ldots & 0 \\
\ldots & \ldots & \ldots & \ldots & \ldots & \ldots & \ldots & \ldots & \ldots & \ldots & \ldots & \ldots & \ldots \\
0 & c_{2 m 1} & \ldots & 0 & 0 & c_{2 m 2} & \ldots & 0 & \ldots & 0 & c_{2 m m} & \ldots & 0 \\
\ldots & \ldots & \ldots & \ldots & \ldots & \ldots & \ldots & \ldots & \ldots & \ldots & \ldots & \ldots & \ldots \\
0 & 0 & \ldots & c_{m 11} & 0 & 0 & \ldots & c_{m 12} & \ldots & 0 & 0 & \ldots & c_{m 1 m} \\
0 & 0 & \ldots & c_{m 21} & 0 & 0 & \ldots & c_{m 22} & \ldots & 0 & 0 & \ldots & c_{m 21} \\
\ldots & \ldots & \ldots & \ldots & \ldots & \ldots & \ldots & \ldots & \ldots & \ldots & \ldots & \ldots & \ldots \\
0 & 0 & \ldots & c_{m m 1} & 0 & 0 & \ldots & c_{m m 2} & \ldots & 0 & 0 & \ldots & c_{m m m}
\end{array}\right)
$$


Так как $L$ обратимо, то матрица $C$ должна быть невырожденной. Заметим, что матрица $C$ разбивается на клетки размеров $m \times m$, а ее строки разбиваются на $m$ полос по $m$ строк в каждой. В каждой клетке матрицы $C$ имеется единственный ненулевой столбец, и система всех ненулевых столбцов каждой полосы линейно независима в силу невырожденности матрицы $C$.

Теорема 1. Если $L-$ блочно-равномерное преобразование, определенное формулами (7) в базисе $E$, то для любых двух множеств $V\left(i_{1}, \ldots, i_{s}\right)$, $V\left(j_{1}, \ldots, j_{t}\right)$ из (1) имеет место равенство

$$
\left|L\left(V\left(i_{1}, \ldots, i_{s}\right)\right) \cap V\left(j_{1}, \ldots, j_{t}\right)\right|=\sum_{k=0}^{t}(-1)^{k} C_{t}^{k}\left(q^{t-k}-1\right)^{s} .
$$

Доказательство. Выразим через базис $E$ произвольные векторы $\alpha \in V\left(i_{1}, \ldots, i_{s}\right), \quad \beta \in V\left(j_{1}, \ldots, j_{t}\right)$ :

$$
\begin{aligned}
& \alpha=\left(a_{11} e_{i_{1} 1}+\ldots+a_{1 m} e_{i_{1} m}\right)+\ldots+\left(a_{s 1} e_{i_{s} 1}+\ldots+a_{s m} e_{i_{s} m}\right), \\
& \beta=\left(b_{11} e_{j_{1} 1}+\ldots+b_{1 m} e_{j_{1} m}\right)+\ldots+\left(b_{t 1} e_{j_{t} 1}+\ldots+b_{t m} e_{j_{t} m}\right),
\end{aligned}
$$

где коэффициенты $a_{i j}, b_{i j}$ удовлетворяют лишь одному условию: в каждой из пар скобок хотя бы один коэффициент отличен от 0. Будем считать $a_{i j}, b_{i j}$ неизвестными и искать их из уравнения

$$
L(\alpha)=\beta .
$$

Выписав подробно вектор $L(\alpha)$ и приравняв коэффициенты при одинаковых базисных векторах в записях векторов $L(\alpha)$ и $\beta$, получим систему уравнений относительно коэффициентов $a_{i j}, b_{i j}$. Опуская чисто технические подробности этого процесса, мы приведем следующий промежуточный результат. Так как в запись вектора $\beta$ могут входить лишь векторы $e_{i j}$ при $i=j_{1}, \ldots, j_{t}$, то все коэффициенты $a_{i j}$, в которых $j \neq j_{1}, \ldots, j_{t}$, необходимо будет приравнять к 0 . Для остальных коэффициентов системы (8) получим $t$ систем линейных уравнений вида

$$
C_{u} A_{u}=B_{u}
$$

относительно неизвестных $a_{i j}, b_{i j}$, где

$$
C_{u}=\left(\begin{array}{cccc}
c_{j_{u}} 1 i_{1} & c_{j_{u} 1 i_{2}} & \ldots & c_{j_{u} 1 i_{s}} \\
c_{j_{u} 2 i_{1}} & c_{j_{u} 2 i_{2}} & \ldots & c_{j_{u} 2 i_{s}} \\
\ldots & \ldots & \ldots & \ldots \\
c_{j_{u} m i_{1}} & c_{j_{u} m i_{2}} & \ldots & c_{j_{u} m i_{s}}
\end{array}\right), A_{u}=\left(\begin{array}{c}
a_{1 j_{u}} \\
a_{2 j_{u}} \\
\ldots \\
a_{s j_{u}}
\end{array}\right), \quad B_{u}=\left(\begin{array}{c}
b_{u 1} \\
b_{u 2} \\
\ldots \\
b_{u m}
\end{array}\right), u=1, \ldots, t,
$$


причем из условия $\beta \in V\left(j_{1}, \ldots, j_{t}\right)$ следует, что нас интересуют лишь такие решения, в которых столбцы $B_{u}$ - ненулевые. Так как множества неизвестных этих систем уравнений попарно не пересекаются, то их можно решать отдельно.

Легко видеть, что столбцы матрицы $C_{u}$ являются ненулевыми столбцами одной и той же полосы матрицы $C$ и потому образуют линейно независимую систему. Следовательно, $\operatorname{rang} C_{u}=s$, и в $C_{u}$ существует $s$ линейно независимых строк, а остальные строки являются их линейными комбинациями. Пусть, например, линейно независимой является система первых $s$ строк. Тогда система (9) будет совместной в том и только том случае, когда последние $m-s$ элементов правой части являются точно такими же линейными комбинациями первых $s$ элементов. Пусть это условие выполнено. Тогда решение системы (9) сведется к решению системы уравнений, полученной из (9) удалением последних $m-s$ уравнений. Запишем эту систему в виде

$$
\bar{C}_{u} A_{u}=\bar{B}_{u} .
$$

Так как матрица $\bar{C}_{u}$ квадратная и невырожденная, то система (10) устанавливает взаимно однозначное соответствие между столбцами $\bar{B}_{u}$ и $A_{u}$, т. е. для любого вектора $A_{u}$ существует единственный вектор $\bar{B}_{u}$, удовлетворяющий условию (10), а потому и единственный вектор $B_{u}$, удовлетворяющий условию (9). При этом нулевому столбцу соответствует нулевой столбец. Проделав описанную процедуру для всех $t$ значений $u$, мы получим $t$ равенств векторов-столбцов, или равенство двух матриц

$$
\left(\bar{C}_{1} A_{1}, \bar{C}_{2} A_{2}, \ldots, \bar{C}_{t} A_{t}\right)=\left(\bar{B}_{1}, \bar{B}_{2}, \ldots, \bar{B}_{t}\right),
$$

которое эквивалентно равенству матриц $A=B$, где

$$
A=\left(A_{1}, A_{2}, \ldots, A_{t}\right), B=\left(\bar{C}_{1}^{-1} \bar{B}_{1}, \bar{C}_{2}^{-1} \bar{B}_{2}, \ldots, \bar{C}_{t}^{-1} \bar{B}_{t}\right) .
$$

Заметим, что если $\bar{B}_{i}$ пробегает множество всех ненулевых столбцов длины $s$, то и $\bar{C}_{i}^{-1} \bar{B}_{i}$ будет пробегать то же самое множество в силу невырожденности матрицы $\bar{C}_{i}^{-1}$. Так как столбцы $B_{u}$ ненулевые, то ненулевыми будут столбцы матриц $B$ и $A$. Кроме того, из включения $\alpha \in V\left(i_{1}, \ldots, i_{s}\right)$ и равенств $a_{i j}=0$ при $j \neq j_{1}, \ldots, j_{t}$ следует, что и все строки матриц $A$ также ненулевые. Следовательно, число искомых векторов $\alpha$ равно числу матриц размеров $s \times t$ над полем $G F(q)$, не содержащих нулевых строк и столбцов. Теперь для завершения доказательства остается воспользоваться известной 
формулой для числа матриц размеров $s \times t$ над полем $G F(q)$ без нулевых строк и столбцов (см. [5], стр. 69). Теорема доказана.

Следствие 2. В условиях теоремы 1 имеем:

а) величина (6) не зависит от чисел $i_{1}, \ldots, i_{s}, j_{1}, \ldots, j_{t}$, а зависит лишь от $s, t$ (обозначим ее значение через $\lambda_{s, t}$ );

b) для чисел $\lambda_{s, t}$ выполняются соотношения $\lambda_{s, t}=\lambda_{t, s} \neq 0$.

ПрИМЕР 1. Пользуясь формулой из теоремы 1 , составим таблицу для чисел $\lambda_{s, t}$ в А-схеме при $q=2, m=3, d=1, r=3$. Занумеруем строки и столбцы таблицы множествами $V(1, \ldots, k), k=1, \ldots, r$, и на пересечении строки с номером $V(1, \ldots, s)$ и столбца с номером $V(1, \ldots, t)$ запишем число $\lambda_{s, t}$. В записях букву $V$ будем опускать.

Таблица 1

$$
\left(\begin{array}{cccccccc}
L & (1) & (2) & (3) & (1,2) & (1,3) & (2,3) & (1,2,3) \\
(1) & 1 & 1 & 1 & 1 & 1 & 1 & 1 \\
(2) & 1 & 1 & 1 & 1 & 1 & 1 & 1 \\
(3) & 1 & 1 & 1 & 1 & 1 & 1 & 1 \\
(1,2) & 1 & 1 & 1 & 7 & 7 & 7 & 25 \\
(1,3) & 1 & 1 & 1 & 7 & 7 & 7 & 25 \\
(2,3) & 1 & 1 & 1 & 7 & 7 & 7 & 25 \\
(1,2,3) & 1 & 1 & 1 & 25 & 25 & 25 & 265
\end{array}\right) .
$$

ПРИМЕР 2. Простейшим примером блочно-равномерного рассеивающего преобразования $L$, определенного формулами вида (7), является преобразование, заданное на базисных векторах формулами

$$
L\left(e_{i j}\right)=e_{j i}, i, j \in\{1, \ldots, r\} .
$$

В этом случае матрица $C$ является подстановочной и потому сравнительно просто реализуется путем постоянной и достаточно просто устроенной перестановки координат векторов из $V_{n}$. Кроме того, она является матрицей 2-го порядка, что также может положительно сказаться на реализации рассматриваемой А-схемы.

В заключение данного пункта найдем еще величины (6) в А-схеме при $m=r d, d=2$. Этот случай также может представлять практический интеpec, например, при $q=2, m=16, r=8, n=128$. 
При $m=r d, d=2$ преобразование $L$ должно отображать из каждого $V(i)$ во все $V(j)$ по 2 базисных вектора. Как и выше, выберем базис

$$
E_{1}=\left\{e_{11}, \ldots, e_{1 m}, e_{21}, \ldots, e_{2 m}, \ldots, e_{r 1}, \ldots, e_{r m}\right\}
$$

и линейное преобразование $L$ в этом базисе зададим формулами

$$
\begin{aligned}
& L\left(e_{i 1}\right)=c_{11}^{(i)} e_{11}+c_{12}^{(i)} e_{12}+\ldots+c_{1 m}^{(i)} e_{1 m}, \quad L\left(e_{i 2}\right)=d_{11}^{(i)} e_{11}+d_{12}^{(i)} e_{12}+\ldots+c_{1 m}^{(i)} e_{1 m}, \\
& L\left(e_{i 3}\right)=c_{21}^{(i)} e_{21}+c_{22}^{(i)} e_{22}+\ldots+c_{2 m}^{(i)} e_{2 m}, L\left(e_{i 4}\right)=d_{21}^{(i)} e_{11}+d_{22}^{(i)} e_{22}+\ldots+c_{2 m}^{(i)} e_{2 m} \text {, } \\
& L\left(e_{i m-1}\right)=c_{r 1}^{(i)} e_{r 1}+c_{r 2}^{(i)} e_{r 2}+\ldots+c_{r m}^{(i)} e_{r m}, \quad L\left(e_{i m}\right)=d_{r 1}^{(i)} e_{r 1}+d_{r 2}^{(i)} e_{r 2}+\ldots+c_{r m}^{(i)} e_{r m},
\end{aligned}
$$

где $i=1, \ldots, r$, а коэффициенты должны выбираться из $G F(q)$ так, чтобы преобразование $L$ было обратимым. Матрица этого преобразования в базисе $E_{1}$, как и выписанная выше матрица $C$, разбивается на клетки размеров $m \times m$. Но число клеток в ней равно $r^{2}$, поскольку ее строки (столбцы) разбиваются на $r$ горизонтальных (вертикальных) полос ширины $\mathrm{m}$. В каждой ее клетке содержится ровно 2 ненулевых столбца. Всего в каждой горизонтальной полосе содержится ровно $m$ ненулевых столбцов, и матрица будет невырожденной тогда и только тогда, когда система ненулевых столбцов каждой полосы будет линейно независимой.

Теорема 2. В А-схеме над полем $G F(q)$ nри $m=2 r$ с биективным преобразованием $L$, определенным формулами (11), для любых двух множеств $V\left(i_{1}, \ldots, i_{s}\right), V\left(j_{1}, \ldots, j_{t}\right)$ из (1) имеет место равенство

$$
\left|L\left(V\left(i_{1}, \ldots, i_{s}\right)\right) \cap V\left(j_{1}, \ldots, j_{t}\right)\right|=\sum_{i=0}^{s} \sum_{j=0}^{t}(-1)^{j} 2^{i} C_{s}^{i} C_{t}^{j}\left(q^{t-j}-1\right)^{2 s-i} .
$$

Доказательство. Из доказательства теоремы 1 следовало, что при $l=1$ число (6) зависит лишь от $s, t$. Аналогичная ситуация имеет место и в рассматриваемом случае. Поэтому будем считать, что $i_{1}=1, \ldots, i_{s}=s$ и $j_{1}=1, \ldots, j_{t}=t$. Тогда любые векторы $\alpha \in V(1, \ldots, s), \beta \in V(1, \ldots, t)$ запишутся в виде

$$
\begin{gathered}
\alpha=\left(a_{11} e_{11}+\ldots+a_{1 m} e_{1 m}\right)+\ldots+\left(a_{s 1} e_{s 1}+\ldots+a_{s m} e_{s m}\right), \\
\beta=\left(b_{11} e_{11}+\ldots+b_{1 m} e_{1 m}\right)+\ldots+\left(b_{t 1} e_{t 1}+\ldots+b_{t m} e_{t m}\right),
\end{gathered}
$$

где сумма в каждой паре скобок отлична от нулевого вектора. Для доказательства теоремы нам необходимо выяснить, при каких коэффициентах $a_{i j}, b_{i j}$ выполняется равенство (8). Рассуждая по той же схеме, что и при до- 
казательстве теоремы 1, мы придем к следующему результату: для любых фиксированных коэффициентов $a_{i j}$ вектора $\alpha$ найдутся требуемые элементы $b_{i j}$ в том и только том случае, когда в матрице

$$
D=\left(\begin{array}{ccccc}
a_{11} & a_{13} & a_{15} & \ldots & a_{12 t-1} \\
a_{12} & a_{14} & a_{16} & \ldots & a_{12 t} \\
a_{21} & a_{23} & a_{25} & \ldots & a_{12 t-1} \\
a_{22} & a_{24} & a_{26} & \ldots & a_{22 t} \\
\ldots & \ldots & \ldots & \ldots & \ldots \\
a_{s 1} & a_{s 3} & a_{s 5} & \ldots & a_{s 2 t-1} \\
a_{s 2} & a_{s 4} & a_{s 6} & \ldots & a_{s 2 t}
\end{array}\right)
$$

размеров $2 s \times t$ над полем $G F(q)$ нет нулевых столбцов. Напомним, что при доказательстве теоремы 1 нам понадобилось находить число матриц заданных размеров без нулевых строк и столбцов. Здесь же не исключаются случаи, когда некоторые строки матрицы $D$ могут быть нулевыми; ненулевыми должны быть лишь конкатенации $i$-й и $(i+1)$-й строк при $i=1,3, \ldots, 2 s-1$. В связи с этим все $2 s$ строк матрицы $D$ разбиваются на $s$ пар строк по указанному принципу. Нахождение числа таких матриц легко сводится к нахождению числа $R(u, v)$ всех матриц заданных размеров $u \times v$ без нулевых строк и столбцов при подходящих значениях $u, v$. Заметим сначала, что при удалении из матрицы $D$ нулевых строк оставшаяся подматрица снова будет матрицей без нулевых столбцов. Кроме того, любая перестановка строк матрицы также не приведет к появлению нулевых столбцов. Следовательно, число матриц $D$ с $i$ нулевыми строками не зависит от того, в каких парах содержатся эти нулевые строки и какая из двух строк соответствующей пары является нулевой. Отсюда получаем:

$$
\left|L\left(V\left(i_{1}, \ldots, i_{s}\right)\right) \cap V\left(j_{1}, \ldots, j_{t}\right)\right|=\sum_{i=0}^{s} 2^{i} C_{s}^{i} R(2 s-i, t) .
$$

Согласно [5] (см. теорему 1.2)

$$
R(u, v)=\sum_{k=0}^{v}(-1)^{k} C_{v}^{k}\left(q^{v-k}-1\right)^{u}=\sum_{k=0}^{u}(-1)^{k} C_{u}^{k}\left(q^{u-k}-1\right)^{v} .
$$

Подставив в (13) первое выражение для $R(2 s-i, t)$ из (14), мы и получим искомую формулу (12). Теорема доказана.

Из доказательства этой теоремы видно, как находить величины (6) при любом $l>2$. 


\section{§ 3. Максимально рассеивающие линейные преобразования}

Выясним, какие значения принимают величины (6) в А-схеме с параметрами $n, m, r, n=m r$, при использовании максимально рассеивающих преобразований. В этом случае мы приведем сначала описание векторов множества $L\left(V\left(i_{1}, \ldots, i_{s}\right)\right) \cap V\left(j_{1}, \ldots, j_{t}\right)$, из которого будут следовать весьма простые оценки величины (6), а затем, используя связи максимально рассеивающих преобразований с МДР-кодами, найдем более громоздкое, но точное ее значение.

Из определения максимально рассеивающего линейного преобразования $L$ видно, что векторы из $V\left(i_{1}, \ldots, i_{s}\right)$ оно может переводить в векторы из $V\left(j_{1}, \ldots, j_{t}\right)$ лишь при $t \geq r+1-s$, в частности, векторы из $V(i)-$ только в $V(1,2, \ldots, r)$, векторы из $V(i, j)$ - только в $V(1,2, \ldots, r)$ и в подмножества вида $V\left(j_{1}, \ldots, j_{r-1}\right)$ и т. д. Вместе с тем, непосредственно из определения не видно, в какие из множеств вида $V\left(j_{1}, \ldots, j_{t}\right)$ и в каком числе фактически переводит $L$ векторы из $V\left(i_{1}, \ldots, i_{s}\right)$ при $t \geq r+1-s$.

Для решения этого вопроса напомним сначала, как обычно строятся максимально рассеивающие преобразования в рассматриваемой ситуации.

Будем рассматривать подблоки блока как элементы поля $G F\left(q^{m}\right)$, записанные в некотором базисе над полем $G F(q)$. Тогда каждый блок будет вектор-строкой длины $r$ с элементами из $G F\left(q^{m}\right)$. Любое линейное преобразование $\phi$ пространства таких строк можно реализовать как умножение векторов на подходящую матрицу $A$ размеров $r \times r$ над полем $G F\left(q^{m}\right): a \phi=a A$. Реализованное таким образом линейное преобразование $\phi$ обозначим через $L_{A}$. В случае, когда $L_{A}-$ максимально рассеивающее преобразование, матрицу $A$ также называют максимально рассеивающей. Всюду далее под максимально рассеивющими преобразованиями будут пониматься лишь преобразования, реализованные указанным образом, хотя, как доказано А. В. Анашкиным, существуют и другие максимально рассеивающие преобразования пространства $V_{n}(q)$.

Обозначим через $A\left(i_{1}, \ldots, i_{s}\right)$ подматрицу максимально рассеивающей матрицы $A$, полученную удалением из $A$ всех ее строк, кроме строк с номерами $i_{1}, \ldots, i_{s}$. Из определения максимально рассеивающего преобразования легко следует, что для любого $s \in\{1, \ldots, r\}$ матрица $A\left(i_{1}, \ldots, i_{s}\right)$ является порождающей для кода длины $r$, размерности $s$ и с максимально достижимым кодовым расстоянием $r-s+1$ (такие коды называют МДР-кодами). Это условие равносильно тому, что любые $s$ столбцов матрицы $A\left(i_{1}, \ldots, i_{s}\right)$ 
линейно независимы (см., например, [3] п. 11.2). Для всей матрицы $A$ это означает, что все ее миноры отличны от нуля. Воспользуемся этим свойством матрицы $A$ для доказательства следующего утверждения.

Теорема 3. Пусть $L=L_{A}-$ максимально рассеивающее преобразование пространства $V_{n}\left(q^{m}\right)$, определенное матрицей А размеров $r \times r$ над полем $G F\left(q^{m}\right)$, и при этом для целых чисел $s, t$ выполнены условия:

$$
1 \leq s \leq r, 1 \leq t \leq r, s+t \geq r+1, r<q^{m}-1 .
$$

Тогда для любых множеств вида $V\left(i_{1}, \ldots, i_{s}\right)$ u $V\left(j_{1}, \ldots, j_{t}\right)$ имеют место неравенства

$$
\left(q^{m}-1\right)^{s+t-r-1}\left(q^{m}-r-1\right) \leq\left|L\left(V\left(i_{1}, \ldots, i_{s}\right)\right) \cap V\left(j_{1}, \ldots, j_{t}\right)\right| \leq\left(q^{m}-1\right)^{s+t-r} .
$$

В частности, если $s+t=r+1$, то $\left|L\left(V\left(i_{1}, \ldots, i_{s}\right)\right) \cap V\left(j_{1}, \ldots, j_{t}\right)\right|=q^{m}-1$.

Доказательство. Для упрощения записей рассмотрим случай, когда $\left(i_{1}, \ldots, i_{s}\right)=(1, \ldots, s)$ и $\left(j_{1}, \ldots, j_{t}\right)=(1, \ldots, t)$. В остальных случаях рассуждения аналогичны. Напомним, что все миноры, а следовательно, и элементы матрицы $A$ отличны от нуля. Для неизвестных векторов $a$ и $b=a L$ введем обозначения:

$$
a=\left(x_{1}, \ldots, x_{r}\right), b=\left(y_{1}, \ldots, y_{r}\right) .
$$

Заметим, что по условию в искомых векторах $a, b$ ненулевыми должны быть первые $s$ и $t$ координат соответственно, остальные координаты должны быть равными 0. Поэтому система уравнений $a A=b$ подробно записывается в следующем виде:

$$
\begin{aligned}
& a_{11} x_{1}+\ldots+a_{s I} x_{s}=y_{1}, \\
& \ldots \ldots \ldots \ldots \ldots \ldots \ldots . \ldots \\
& a_{1 t} x_{1}+\ldots+a_{s t} x_{s}=y_{t}, \\
& a_{1 t+1} x_{1}+\ldots+a_{s t+1} x_{s}=0, \\
& \ldots \ldots \ldots \ldots \ldots \ldots \ldots \\
& a_{1 r} x_{1}+\ldots+a_{s r} x_{s}=0 .
\end{aligned}
$$

Наша цель заключается в нахождении оценок для числа решений этой системы, не содержащих нулевых компонент. Замечаем, что это есть однородная система $r$ уравнений с $s+t$ неизвестными $x_{1}, \ldots, x_{s}, y_{1}, \ldots, y_{t}$. Легко видеть, что ранг основной матрицы этой системы равен $r$. Если $t=r$, то из условия $a_{s j} \neq 0, j=1, \ldots, r$, следует, что при любых ненулевых значениях $x_{1}, \ldots, x_{s-1}$ найдется не менее $q^{m}-r-1$ ненулевых значений для $x_{s}$, при которых значения $y_{1}, \ldots, y_{r}$ будут отличными от 0. Значит, в этом случае утверждение 
теоремы верно. Пусть $t<r$. Объявим неизвестные $x_{1}, \ldots, x_{r-t}, y_{1}, \ldots, y_{t}$ связанными и перенесем соответствующие члены уравнений в левые части, а неизвестные $x_{r-t+1}, \ldots, x_{s}-$ свободными, и содержащие их члены перенесем в правые части. Пусть $s+t>r+1$. Тогда неизвестным $x_{r-t+1}, \ldots, x_{s-1}$ придадим произвольные ненулевые значения из $G F\left(q^{m}\right)$. В итоге получим систему из $r$ уравнений с $r$ неизвестными и с одним свободным параметром $x_{s}$. Нетрудно видеть, что определитель $\Delta$ этой системы с точностью до знака совпадает с минором порядка $r-t$ матрицы $A$, и потому $\Delta \neq 0$, правые части системы имеют вид $b_{i} \oplus a_{s i} x_{s}$. Решая эту систему по формулам Крамера, получим:

$$
x_{i}=\Delta^{-1}\left(u_{i} \oplus c_{i} x_{s}\right), \mathrm{i}=1, \ldots, r-t, y_{j}=\Delta^{-1}\left(v_{j} \oplus d_{j} x_{s}\right), j=1, \ldots, t,
$$

где $c_{i}, d_{j}$ с точностью до знака совпадают с минорами матрицы $A$ соответственно порядков $r-t u r-t+1$ и, значит, отличны от 0 . Так как $q^{m}-1>r$, то найдется такое ненулевое значение неизвестного $x_{s}$, при котором значения неизвестных $x_{1}, \ldots, x_{r-t}, y_{1}, \ldots, y_{t}$ будут также ненулевыми. Причем таких значений для $x_{s}$ найдется не более $q^{m}-1$ и не менее $q^{m}-1-r$ при любых ненулевых значениях неизвестных $x_{r-t+1}, \ldots, x_{s-1}$. Следовательно, при $s+t>r+1$ теорема верна. Если же $s+t=r+1$, то свободным неизвестным является лишь $x_{s}$, и в этом случае элементы $u_{i}, v_{j}$ будут нулевыми. Тогда значения неизвестных $x_{i}, i=1, \ldots, r-t, y_{j}, j=1, \ldots, t$, будут ненулевыми при любом ненулевом значении $x_{s}$. Следовательно, в этом крайнем случае число искомых векторов в точности равно $q^{m}-1$. Теорема доказана.

Для нахождения точного значения величин (6) нам понадобится одно вспомогательное утверждение А. А. Нечаева о векторах заданного веса (т. е. с заданным числом ненулевых компонент) для любых МДР-кодов, представляющее и самостоятельный интерес в теории кодирования. Так как этот результат, по-видимому, не опубликован, то приведем его доказательство, ограничившись для нашей цели линейными МДР-кодами.

Лемма. Пусть $K(r, s)$ - линейный МДР-код над конечным полем $P$ длины $r$ размерности s с кодовым расстоянием $d u t \in\{d, d+1, \ldots, r\}$. Тогда в $K(r, s)$ число векторов веса $t$ с ненулевыми элементами на t фиксированных позициях не зависит от выбора номеров позичий, а однозначно определяется числами $r, s, t$.

Доказательство. Для простоты записей будем рассматривать случай, когда фиксированными являются первые $t$ мест. В остальных случаях рассуждения аналогичны. Обозначим через $K_{t}(r, s)$ множество кодовых слов кода $K(r, s)$ с нулями на последних $r-t$ позициях и через $\bar{K}_{t}(r, s)-$ 
множество векторов длины $t$, полученных из векторов множества $K_{t}(r, s)$ удалением $r-t$ последних компонент. Докажем, что пространство $\bar{K}_{t}(r, s)$ есть МДР-код длины $t$ и размерности $s_{1}=s-(r-t)$. (Заметим, что $s-(r-t)>0$ в силу условия $d \leq t \leq r$.) Очевидно, что $\operatorname{dim} \bar{K}_{t}(r, s) \leq s_{1}$. Поэтому достаточно доказать, что в матрице $C$, составленной из строк кода $\bar{K}_{t}(r, s)$, любые $s_{1}$ столбцов линейно независимы (см. следствие 2 теоремы 2 п. 11.2 из [4]). Для простоты возьмем первые $s_{1}$ столбцов и покажем, что в $C$ найдется строка с любым набором элементов поля $P$. Так как $s_{1}+r-t=s$, то в исходном коде $K(r, s)$ для любых $a_{1}, \ldots, a_{s_{1}}$ найдется вектор $\left(a_{1}, \ldots, a_{s_{1}}, a_{s_{1}+1}, \ldots, a_{t}, 0, \ldots, 0\right)$ при некоторых $a_{s_{1}+1}, \ldots, a_{t}$, а тогда в $\bar{K}_{t}(r, s)$ встретится вектор $\left(a_{1}, \ldots, a_{s_{1}}, a_{s_{1}+1}, \ldots, a_{t}\right)$. Следовательно, система выбранных столбцов линейно независима, и $\bar{K}_{t}(r, s)$ есть МДР-код. Из его построения видно, что множества векторов веса $t$ с ненулевыми компонентами на первых $t$ местах в кодах $K(r, s), \bar{K}_{t}(r, s)$ совпадают. И в то же время это множество векторов совпадает с множеством всех векторов веса $t$ кода $\bar{K}_{t}(r, s)$. Однако в любом МДР-коде множество векторов любого фиксированного веса $w$ определяется основными параметрами кода и числом $w$ (см. теорему 6 п. 11.3 из [4]). Это и доказывает утверждение леммы.

Теорема 4. Если выполнены условия теоремы 3, то

$$
\left|L\left(V\left(i_{1}, \ldots, i_{s}\right)\right) \cap V\left(j_{1}, \ldots, j_{t}\right)\right|=\left(q^{m}-1\right) \sum_{j=0}^{s+t-(r+1)}(-1)^{j} C_{s+t-1}^{j} q^{m(s+t-(r+j+1))} .
$$

Доказательство. Рассмотрим МДР-код $K\left(i_{1}, \ldots, i_{s}\right)$ длины $r$ и размерности $s$ над полем $P=G F\left(q^{m}\right)$ с порождающей матрицей $A\left(i_{1}, \ldots, i_{s}\right)$ :

$$
K\left(i_{1}, \ldots, i_{s}\right)=\left\{\left(x_{1}, \ldots, x_{s}\right) A\left(i_{1}, \ldots, i_{s}\right): x_{1}, \ldots, x_{s} \in G F\left(q^{m}\right)\right\},
$$

и обозначим множество всех его векторов веса $t$ через $K_{t}\left(i_{1}, \ldots, i_{s}\right)$. Так как кодовое расстояние $d=r-s+1$ и по условию $s+t \geq r+1$, то $t \geq d$, и потому множество $K_{t}\left(i_{1}, \ldots, i_{s}\right)$ не пусто. Пусть $K_{t}\left(i_{1}, \ldots, i_{s}, j_{1}, \ldots, j_{t}\right)$ есть подмножество всех его векторов с ненулевыми компонентами на местах с номерами $j_{1}, \ldots, j_{t}$. По теореме 6 п. 11.3 из [3]

$$
\left|K_{t}\left(i_{1}, \ldots, i_{s}\right)\right|=C_{r}^{t}\left(q^{m}-1\right) \sum_{j=0}^{s+t-(r+1)}(-1)^{j} C_{t-1}^{j} q^{m(s+t-(r+1+j))} .
$$


Согласно лемме величина $K_{t}\left(i_{1}, \ldots, i_{s}, j_{1}, \ldots, j_{t}\right)$ не зависит от выбора чисел $j_{1}, \ldots, j_{t}$. Поэтому

$$
\left|K_{t}\left(i_{1}, \ldots, i_{s}, j_{1}, \ldots, j_{t}\right)\right|=\left(q^{m}-1\right) \sum_{j=0}^{s+t-(r+1)}(-1)^{j} C_{t-1}^{j} q^{m(s+t-(r+1+j))} .
$$

Из последней формулы видно, что число $\left|K_{t}\left(i_{1}, \ldots, i_{s}, j_{1}, \ldots, j_{t}\right)\right|$ не зависит от $i_{1}, \ldots, i_{s}, j_{1}, \ldots, j_{t}$, а зависит лишь от $s$ и $t$, причем $s$ и $t$ входят в нее симметричным образом. Обозначим это число через $v(s, t)$. Теперь для нахождения искомой величины нам нужно удалить из $K_{t}\left(i_{1}, \ldots, i_{s}, j_{1}, \ldots, j_{t}\right)$ все векторы, которые определяются формулой (16) при наборах $\left(x_{1}, \ldots, x_{s}\right)$, содержащих нулевые координаты, и найти число оставшихся векторов. Множество всех подлежащих удалению векторов можно представить в виде объединения множеств $U_{1}, \ldots, U_{s}$, где $U_{i}$ соответствует наборам векторов $\left(x_{1}, \ldots, x_{s}\right)$, в которых $x_{i}=0$, а остальные компоненты произвольные. Очевидно, что $U_{i}$ есть МДР-код длины $r$ и размерности $s-1$. Его порождающая матрица получается удалением из матрицы $A\left(i_{1}, \ldots, i_{s}\right)$ ее $i$-й строки. По формуле включения-исключения получаем:

$$
\left|U_{1} \cup \ldots \cup U_{s}\right|=C_{s}^{s-1} v(s-1, t)-C_{s}^{s-2} v(s-2, t)+\ldots+(-1)^{s-2} v(1, t) .
$$

Отсюда видно, что искомая величина $\left|L\left(V\left(i_{1}, \ldots, i_{s}\right)\right) \cap V\left(j_{1}, \ldots, j_{t}\right)\right|$ также зависит лишь от $s$ и $t$. Обозначим ее через $\mu(s, t)$. Из проведенных рассуждений видно, что

$$
\mu(s, t)=v(s, t)-C_{s}^{s-1} v(s-1, t)+C_{s}^{s-2} v(s-2, t)-\ldots+(-1)^{s-1} v(1, t) .
$$

Заменив в последней формуле величины $v(j, t)$ их найденными выше значениями при $j=1, \ldots, s$ и воспользовавшись равенствами

$$
\sum_{i=0}^{k} C_{u}^{i} C_{v}^{k-i}=C_{u+v}^{k}, \quad k \leq u, k \leq v,
$$

получим искомую формулу (15). Теорема доказана.

Следствие 3. В условиях теорем 3-4 для чисел $\mu_{s, t}$ выполняются следуюшие соотношения:

1) $\mu_{s, t}=\mu_{t, s}$, 2) $\left.\mu_{s, t}>0 \Leftrightarrow s+t \geq r+1,3\right) \mu_{s, t}=q^{m}-1$, если $s+t=r+1$.

Следствие 4. Если в $A_{1}$-схеме подстановки $\Pi_{i}$ выбраны так, что $\left(Q_{i}\right)^{2}>0, \quad i \in\{1, \ldots, r\}$, а преобразования $L_{2}, L_{4}$ - максимально рассеивающие, то матрица $\left(Q^{(\Pi)}\right)^{2} \tilde{L}_{2}\left(Q^{(\Pi)}\right)^{2}$ не является положительной, а

$$
\left(Q^{(\Pi)}\right)^{2} \tilde{L}_{2}\left(Q^{(\Pi)}\right)^{2} \tilde{L}_{4} Q^{(\Pi)}>0,
$$


m. е. минимальное число раундов $N$, за которое $A_{1}$-схемой реализуется 2транзитивное множество подстановок, равно 6, причем на 6-м раунде требуется лишь прибавление ключей.

Доказательство. Первое утверждение следует из утверждения 1, если учесть, что $\mu_{s, t}=0$ при $s+t<r+1$. Докажем неравенство (17). Из теоремы 4 и утверждения 1 следует, что в матрице $\left(Q^{(\text {П) }}\right)^{2} \tilde{L}_{2}\left(Q^{(\text {П) }}\right)^{2}$ в строках с номерами $V\left(i_{1}, \ldots, i_{s}\right)$ будут ненулевыми все элементы из столбцов с номерами $V\left(j_{1}, \ldots, j_{t}\right)$ при всех $t \geq r-s+1$ и любых $j_{1}, \ldots, j_{t}$. Далее, применяя теорему 4 к матрице, транспонированной к $Q^{(\text {П) }}$, замечаем, что в матрице $\tilde{L}_{4} Q^{(\Pi)}$ в строках с номерами $V\left(j_{1}, \ldots, j_{t}\right)$ при всех $t \geq r-s+1$ и любых $j_{1}, \ldots, j_{t}$ в каждом столбце есть ненулевые элементы. Отсюда следует, что в строках с номерами из $V\left(i_{1}, \ldots, i_{s}\right)$ матрицы $\left(Q^{(\text {П) }}\right)^{2} \tilde{L}_{2}\left(Q^{(\text {(ा) }}\right)^{2} \tilde{L}_{4} Q^{(I)}$ все элементы отличны от нуля. Так как это верно для любых множеств $V\left(i_{1}, \ldots, i_{s}\right)$ из (1), то неравенство (19) доказано.

ЗАМЕЧАНИЕ 1 . В статье Ф. М. Малышева [3] оценивалось число $N$ раундов, необходимое для реализации 2-транзитивного множества подстановок XSL-сетями, в которых подблоками были элементы поля $F$, в качестве под-

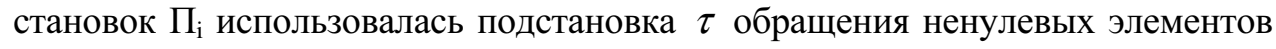
поля $F$, к блокам в нечетных раундах применялись мономиальные матрицы, а в четных - матрицы с отличными от нуля элементами и их алгебраическими дополнениями. При этом, естественно, возникает вопрос о сохранении полученных оценок показателей 2-транзитивности при замене подстановки $\tau$ любыми подстановками $\Pi_{\mathrm{i}}$, удовлетворяющими условию $\left(Q^{\left(\Pi_{i}\right)}\right)^{2}>0$ (которое выполняется при $\Pi_{i}=\tau$ ). На этот вопрос в случае конечного поля $F$ отвечает следующее утверждение, которое существенно обобщает и частично улучшает результаты из [3].

Утверждение 3. Если в $A_{1}$-схеме из следствия 2 заменить тождественные преобразования $L_{1}, L_{3}$ умножением на мономиальные матрищы, амаксимально рассеивающие преобразования $L_{2}, L_{4}-$ умножением на матрицы с неравными нулю минорами 1-го и $(r-1)$-го порядков, то для полученной XSL-сети будет выполняться соотношение

$$
\left(Q^{(\Pi)}\right)^{2} \tilde{L}_{2}\left(Q^{(\Pi)}\right)^{2} \tilde{L}_{4}\left(Q^{(I)}\right)^{2}>0 .
$$

Если при этом число $q^{m}-r-1$ больше максимального числа нулей по столбиам матрии $Q_{i}, i \in\{1, \ldots, r\}$, то выполняется неравенство (17).

Иначе говоря, 2-транзитивное множество подстановок реализуется в 1-м случае за 7, а во 2-м случае - за 6 раундов. 
Доказательство. Возможность указанной замены $L_{1}, L_{3}$ с сохранением оценок объясняется тем, что матрица $Q^{(П S)} Q^{(П)}$, как и $\left(Q^{(П)}\right)^{2}$, состоит из положительных клеток тех же размеров, но расположенных несколько иначе (в соответствии с перестановкой $S$ ). Заменим еще $L_{2}, L_{4}$ на указанные в утверждении 3 преобразования. Из доказательства теоремы 3 видно, что при $t=r$ и любом $s$ требовалось лишь, чтобы отличными от нуля были элементы матрицы $A, a$ при $s=r$ и любом $t$ - чтобы отличными от нуля были все миноры порядка $r-1$ матрицы $A$. Таким образом, неравенства из теоремы 3 при $s=r$ и любом $t$, а также $t=r$ и любом $s$ останутся верными при указанной замене $L_{2}, L_{4}$. Следовательно, по утверждению 1 в матрице $\left(Q^{(\Pi)}\right)^{2} \tilde{L}_{2}\left(Q^{(\text {П) }}\right)^{2}$ будут отличными от нуля все элементы столбцов и строк с номерами из $V(1,2, \ldots, r)$, а в матрице $\tilde{L}_{4}\left(Q^{(П)}\right)^{2}$ в системе строк с номерами из $V(1,2, \ldots, r)$ каждый столбец будет содержать ненулевые элементы. Отсюда и из правила умножения матриц следует, что неравенство (18) выполнено. Теперь для доказательства неравенства (17) достаточно показать, что в матрице $\tilde{L}_{4} Q^{(\text {() }}$ в системе всех строк с номерами из $V(1, \ldots, r)$ каждый столбец будет содержать хотя бы один ненулевой элемент. Для простоты записей рассмотрим столбец с номером $b=\left(b_{1}, \ldots, b_{r}\right) \in V(1, \ldots, s)$. Согласно теореме 3 в строки с номерами из $V(1, \ldots, r)$ под действием $L_{4}$ перейдут все строки с номерами вида $\left(x_{1}, \ldots, x_{s}, 0, \ldots, 0\right) \in V(1, \ldots, s)$, где $x_{1}, \ldots, x_{s-1}$ - любые, отличные от 0 , а $x_{s}$ принимает не менее $q^{m}-r-1$ значений при любых фиксированных $x_{1}, \ldots, x_{s-1}$. Зафиксируем $x_{1}, \ldots, x_{s-1}$ так, чтобы выполнялись условия $q_{x_{i}, b_{i}}^{(i)} \neq 0, i=1, \ldots, s-1$. Так как $\left(q^{m}-r-1\right)>p$, то для указанных $i=1, \ldots, s-1$ найдется значение $x_{s}$, при котором $q_{x_{s}, b_{s}}^{(s)} \neq 0$. Отсюда, учитывая, что $Q_{1, \ldots, s}=Q_{1} \otimes \ldots \otimes Q_{s}$, получаем: в столбце с номером $b$ матрицы $Q_{1, \ldots, s}$ найдется ненулевой элемент, который перейдет в строку с номером из $V(1, \ldots, r)$. Следствие 3 доказано.

ПРИМЕР 3. Пользуясь формулой (17), составим таблицу для чисел $\mu_{s, t}$ в $A$-схеме при $q=2, n=9, m=3, d=1$ в случае, когда $L_{2}, L_{4}$ совпадают с максимально рассеивающим преобразованием $L_{A}$, определяемом матрицей

$$
A=\left(\begin{array}{lll}
1 & 2 & 1 \\
2 & 1 & 1 \\
3 & 1 & 2
\end{array}\right)
$$


над полем $G F\left(2^{3}\right)$. В этом случае таблица имеет следующий вид:

Таблица 2

$\left(\begin{array}{cccccccc}L & (1) & (2) & (3) & (1,2) & (1,3) & (2,3) & (1,2,3) \\ (1) & 0 & 0 & 0 & 0 & 0 & 0 & 7 \\ (2) & 0 & 0 & 0 & 0 & 0 & 0 & 7 \\ (3) & 0 & 0 & 0 & 0 & 0 & 0 & 7 \\ (1,2) & 0 & 0 & 0 & 7 & 7 & 7 & 28 \\ (1,3) & 0 & 0 & 0 & 7 & 7 & 7 & 28 \\ (2,3) & 0 & 0 & 0 & 7 & 7 & 7 & 28 \\ (1,2,3) & 7 & 7 & 7 & 28 & 28 & 28 & 238\end{array}\right)$

\section{§ 4. Составные рассеивающие преобразования}

Рассмотрим А-схему с блоками длины $n=m r, r$ подблоками длины $m$, в которой $r=d h, d>1, h>1$, и подстановка П устроена так же, как и в Асхемах из $\S 2,3$. Для рассматриваемых ниже вопросов, не теряя общности, можно считать, что на всех раундах действует одно и то же рассеивающее составное линейное преобразование $L$. Оно представляется в виде произведения $L=\hat{g} f$, где $\widehat{g}$ - пока любая перестановка подблоков, индуцируемая подстановкой $g$, действующей на множестве $\{1, \ldots, r\}$ всех номеров подблоков, а $f$ определяется следующим образом.

Каждый блок $\alpha$ представляется в виде конкатенации $h$ подвекторов длины $m d$ :

$$
\alpha=\left(\alpha_{1}, \alpha_{2}, \ldots, \alpha_{h}\right) .
$$

Вектор $\alpha_{i}$ условимся называть $i$-м каскадом блока $\alpha$. Подчеркнем, что каскад $\alpha_{i}$ является конкатенацией всех подблоков с номерами

$$
(i-1) d+1,(i-1) d+2, \ldots, i d, \quad i=1,2, \ldots, h .
$$

Пусть $U_{i}-$ множество $i$-х каскадов всех блоков; является линейным пространством размерности $m d$ над полем $G F(q)$, и $f$ задается системой линейных преобразований $f_{1}, \ldots, f_{h}$, действующих соответственно на пространствах $U_{1}, \ldots, U_{h}$. Так что для вектора (19)

$$
f(\alpha)=\left(f_{l}\left(\alpha_{1}\right), f_{2}\left(\alpha_{2}\right), \ldots, f_{h}\left(\alpha_{h}\right)\right) .
$$

В данном параграфе мы будем рассматривать лишь $\mathrm{A}_{1}$-схемы с составными рассеивающими преобразованиями в четных раундах, причем все 
$f_{1}, \ldots f_{h}$ будем считать либо максимально рассеивающими, либо блочноравномерными преобразованиями, действующими на соответствующих пространствах. Кроме того, будем предполагать, что квадраты диагональных клеток $Q_{i}$ матрицы $Q^{(\text {П) }}$ положительны.

Введем еще следующие обозначения:

если $\left\{i_{1}, \ldots, i_{s}\right\}=M$, то $V\left(i_{1}, \ldots, i_{s}\right)=V(M) \quad$ и $N(V(M))=M$ для любого $V\left(i_{1}, \ldots, i_{s}\right)$ из $(1)$

$W\left(i_{1}, \ldots, i_{k}\right), 1 \leq i_{1}<\ldots<i_{k} \leq d,-$ множество всех блоков, в которых каскады с номерами $i_{1}, \ldots, i_{k}$ ненулевые, а остальные каскады нулевые, в частности, $W(i)$ состоит из всех подмножеств $V\left(j_{1}, \ldots, j_{s}\right)$, где $1 \leq s \leq d$, a $\left(j_{1}, \ldots, j_{s}\right)$ - любое сочетание из чисел $(20)$;

$\bar{W}(i)=V((i-1) d+1,(i-1) d+2, \ldots, i d) \quad$ и $N(\bar{W}(i))$ есть множество чисел (20);

$C\left(\bar{W}\left(i_{1}\right) \cup \ldots \cup \bar{W}\left(i_{t}\right)\right)$ - замыкание множества $\bar{W}\left(i_{1}\right) \cup \ldots \cup \bar{W}\left(i_{t}\right)$, т. е. система множеств

$$
\left\{V\left(R_{1} \cup \ldots \cup R_{t}\right): \varnothing \neq R_{s} \subset N\left(\bar{W}\left(i_{s}\right)\right), s=1, \ldots, t\right\} .
$$

Множество чисел вида $N\left(\bar{W}\left(i_{1}\right)\right) \cup \ldots \cup N\left(\bar{W}\left(i_{t}\right)\right)$, где $1 \leq i_{1}<\ldots<i_{t} \leq h$, $1 \leq t \leq h$, назовем полно-каскадным, а при $t<h-$ собственным полнокаскадным.

При рассмотрении указанной А-схемы естественно возникает вопрос о выборе подстановки $g$, при которой матрица $\left(Q^{(П)}\right)^{2} \tilde{L}$ сходится к положительной, а значит, и к равномерной матрице. В следующем утверждении дается необходимое и более сильное достаточное условие на $g$.

Теорема 5. Пусть в $A_{1}$-схеме используется составное рассеивающее преобразование $L=\hat{g} f$, где $\hat{g}$ - перестановка подблоков, индуиируемая перестановкой g номеров подблоков, преобразования $f_{1}, \ldots, f_{h}$ все являются или максимально рассеивающими, или блочно-равномерными, и $\left(Q_{i}\right)^{2}>0$, $i=1, \ldots, r$.

Тогда матрииа $\left(Q^{(\Pi)}\right)^{2} \tilde{L}$ сходится $\kappa$ равномерной матрице в том и только том случае, когда в группе $<g>$ отсутствует область импримитивности, являющаяся собственным полно-каскадным множеством.

Заметим, что с определением области импримитивности (или блока) группы подстановок и с простейшими свойствами блоков можно познакомиться, например, по книге [7] (см. также [2]).

Доказательство. Матрица $\left(Q^{(\Pi)}\right)^{2} \tilde{L}$ сходится к равномерной матрице в том и только том случае, когда при некотором $p$ матрица $\left(\left(Q^{(\Pi)}\right)^{2} \tilde{L}\right)^{p}$ положительна. Из утверждения 1 следует, что последнее условие выполнено 
лишь в том случае, когда в каждой строке матрицы $\left(\left(Q^{(\Pi)}\right)^{2} \tilde{L}\right)^{p-1}$ найдется хотя бы один ненулевой элемент в столбцах с номерами каждого из множеств (1). В связи с этим, необходимо проследить, в какие из множеств (1) будут переходить векторы заданного множества $V\left(i_{1}, \ldots, i_{s}\right)$ под действием раундовых преобразований. Напомним, что подстановки $\sigma_{x}$ не изменяют разностей блоков, подстановки $\Pi_{i}$ действуют независимо на множествах $V(i)$ при $i=1, \ldots, r$, а преобразование $L$ переставляет номера столбцов матрицы $\left(Q^{(I)}\right)^{2}$ на втором раунде, матрицы $\left(Q^{(I)}\right)^{2} \tilde{L}\left(Q^{(I I)}\right)^{2}$ на четвертом раунде и т. д. Из всего сказанного следует, что для решения вопроса об условиях и скорости сходимости матрицы $\left(Q^{(П)}\right)^{2} \tilde{L}$ к положительной матрице достаточно следить лишь за действиями преобразования $\tilde{L}$. Условимся каждое применение к матрице преобразования $\tilde{L}$ с последующим умножением на матрицу $\left(Q^{(\Pi)}\right)^{2}$ называть шагом работы схемы. Кроме того, достаточно проследить лишь за векторами множеств $V(j), j=1, \ldots, h$, т. е. за ненулевыми векторами отдельных подблоков, так как если векторы из них удастся перевести во все множества из (1), то, очевидно, то же самое будет выполняться и для векторов из $V\left(i_{1}, \ldots, i_{s}\right)$ при $s>1$.

Рассмотрим сначала случай, когда $f_{i}$ - максимально рассеивающие преобразования.

Зафиксируем в матрице $\left(Q^{(I)}\right)^{2}$ строку с номером из $V(j)$. По условию ненулевыми элементами в этой строке будут все элементы из столбцов с номерами из $V(j)$. Пользуясь свойством максимально рассеивающего преобразования, указанным в теореме 3 , и утверждением 1 , проследим за переходами номеров столбцов из множества $V(j)$.

Шаг 1. Под действием подстановки $\hat{g}$ множество $V(j)$ перейдет в множество $V(g(j))$, которое, очевидно, содержится в единственном из множеств $W(1), \ldots, W(h)$. Если оно содержится в $W(i)$, то после 1-го шага в строках с номерами из $V(j)$ матрицы $\left(Q^{(\Pi)}\right)^{2} \tilde{L}\left(Q^{(\Pi)}\right)^{2}$ ненулевыми будут все элементы столбцов с номерами из полно-каскадного множества $\bar{W}(i)$. Обозначим $N(\bar{W}(i))=M_{1}$.

Шаг 2. Под действием преобразования $\hat{g}$ множество $\bar{W}(i)=V\left(M_{1}\right)$ перейдет в $V\left(g\left(M_{1}\right)\right)$. Для нахождения образов векторов из множества $V\left(g\left(M_{1}\right)\right)$ под действием $f$ необходимо найти все те множества $W(s), s=1, \ldots, h$, в которые переходят некоторые подблоки из $V\left(g\left(M_{1}\right)\right)$, т. е. для которых выполняется условие

$$
g\left(M_{1}\right) \cap N(\bar{W}(s)) \neq \varnothing .
$$


Пусть для определенности это условие выполняется при $s=1, \ldots, t$, причем $g\left(M_{1}\right) \cap N(\bar{W}(s))=M_{1, s}$. Тогда векторы из $V\left(M_{1, s}\right)$ под действием $f$ перейдут в некоторые множества из (1), содержащиеся в $W(s)$. Эти множества описаны в теоремах 3-4. Среди них максимальным по рангу будет множество $\bar{W}(s)$. Отсюда следует, что под действием $f$ множество $V\left(g\left(M_{1}\right)\right)$ перейдет в некоторые подмножества вида $V(M)$, из которых максимальным по рангу будет множество $V\left(M_{2}\right)$, где

$$
M_{2}=N(\bar{W}(1)) \cup, \ldots, \cup N(\bar{W}(t)) .
$$

Шаг 3. Здесь аналогичным образом будем находить все множества из (1), в которые будут переходить векторы из $V(g(M))$ для всех $M$, полученных на 2-м шаге.

Продолжая этот процесс и выписывая из получаемых на каждом шаге множеств лишь максимальные по рангу, получим последовательность множеств

$$
\mathrm{M}_{1}, \mathrm{M}_{2}, \mathrm{M}_{3}, \ldots
$$

Из ее построения видно, что каждое из множеств $M_{i}$ является полнокаскадным,

$$
\left|\mathrm{M}_{1}\right| \leq\left|\mathrm{M}_{2}\right| \leq\left|\mathrm{M}_{3}\right| \leq \ldots
$$

и если $M_{c}=M_{d}$, то $M_{c+1}=M_{d+1}$. Отсюда следует, что последовательность (21) является смешанной периодической с некоторым минимальным периодом

$$
M_{c}, \ldots, M_{c-1+T} .
$$

При этом, очевидно, будут выполняться равенства $M_{c+b} \subseteq g\left(M_{c+b-1}\right)$, $b=1, \ldots, T-1$. Отсюда и из (22) следует, что $M_{c+b}=g\left(M_{c+b-1}\right), b=1, \ldots, T-1$.

Пусть группа $<g>$ имеет областью импримитивности собственное полно-каскадное множество $M$. Тогда, применяя к $V(j)$ при $j \in M$ описанный выше процесс, мы после каждого шага будем получать полно-каскадное множество, содержащееся в области импримитивности, сопряженной с $M$. Следовательно, будем иметь

$$
M_{c} \neq\{1, \ldots, r\} .
$$

Отсюда видно, что при любом натуральном $x$ элементы матрицы $\left(\left(Q^{(I)}\right)^{2} \tilde{L}\right)^{x}$ в строках с номерами из $V(j)$ и столбцах с номерами из $V(1, \ldots, r)$ будут нулями, и значит, матрица $\left(Q^{(I T)}\right)^{2} \tilde{L}$ не будет сходиться к равномерной матрице.

Пусть группа $<g>$ не имеет области импримитивности, являющейся собственным полно-каскадным множеством. Допустим, что при некотором $j \in\{1, \ldots, r\}$ будет выполняться неравенство (24). Если период $T=1$, то $M_{c}$ 
является собственным полно-каскадным инвариантным подмножеством группы $<g>$. Если $T>1$ и множества из (23) попарно не пересекаются, то они являются нетривиальными полно-каскадными областями импримитивности группы $<g>$. Пусть $T>1$ и какие-либо два множества из (23) имеют непустое пересечение, например, $M_{c} \cap M_{c+i}=B_{1 \neq} \varnothing$. Тогда по последовательности (23) можно построить периодическую последовательность с минимальным периодом

$$
B_{1}, B_{2}, \ldots, B_{S},
$$

в которой $S$ делит $T, g\left(B_{m}\right)=B_{m+1},\left|B_{1}\right|<\left|M_{c}\right|$, и каждое из множеств $B_{i}$ является полно-каскадным. Если $s=1$ или $s>1$ и множества из (25) попарно не пересекаются, то они являются областями импримитивности группы $\langle g\rangle$. В противном случае из них аналогичным образом можно будет построить новую периодическую последовательность множеств еще меньшей мощности. Продолжая этот процесс, мы придем к периодической последовательности с одним множеством или с попарно не пересекающимися множествами. Таким образом, во всех случаях получаем нетривиальную полно-каскадную область импимитивности группы $<g>$, что противоречит условию. Следовательно, для любого $j \in\{1,2, \ldots, r\}$ найдется такое $c$, что

$$
M_{c}=\{1, \ldots, r\} .
$$

Не теряя общности, можно считать, что $c-$ одно и то же для всех $j$. В противном случае можно было бы взять наибольшее из них. Наша цель заключается в том, чтобы на некотором раунде получить все множества из (1).

Из (26) следует, что на шаге с номером $c$ получено множество $\bar{W}(1) \bigcup \ldots \bigcup \bar{W}(h)$. Тогда по свойству максимально рассеивающих преобразований, доказанному в теоремах $3-4$, мы на $(c+1)$-м шаге получим замыкание $C(\bar{W}(1) \bigcup \ldots \bigcup \bar{W}(h))$.

Возьмем теперь произвольное полно-каскадное множество $M$ порядка $d(h-1)$ и найдем множество $g^{-1}(M)$. Пусть для простоты записей

$$
M=N\left(\bar{W}_{1}\right) \cup \ldots \cup N\left(\bar{W}_{h-1}\right) .
$$

Если для некоторого $i \in\{1, \ldots, h\}$ имеет место равенство $M \cap g(N(\bar{W}(i)))=\varnothing$, то $g(N(\bar{W}(i)))=N(\bar{W}(i))$, что противоречит условию на группу $\langle g>$. Следовательно, $g^{-1}(M) \bigcap N(\bar{W}(i)) \neq \varnothing$ при $i=1, \ldots, h$, и потому $V\left(g^{-1}(M)\right)$ содержится в $C(\bar{W}(1) \cup \ldots \cup \bar{W}(h))$. Значит, множество $V\left(g^{-1}(M)\right)$ получено на $(c+1)$-м шаге, а на $(c+2)$-м шаге оно под действием подстановки $g$ перейдет в $V(M)$, из которого под действием $f$ получим замыкание множества $M$. Аналогично будут получены замыкания для всех полно-каскадных мно- 
жеств порядка $d(h-1)$. Заметим, что при этом сохранится и множество $\bar{W}(1) \bigcup \ldots \bigcup \bar{W}(h)$, поскольку $g\{1,2, \ldots, r\}=\{1,2, \ldots, r\}$. Аналогично, на $(c+3)$-м шаге мы из замыканий полно-каскадных множеств порядка $d(h-1)$ получим замыкания всех полно-каскадных множеств порядка $d(h-2)$, сохранив полученные ранее замыкания полно-каскадных множеств порядков $d h$ и $d(h-1)$. Продолжая этот процесс, мы после $(c+h)$-го шага будем иметь замыкания всех полно-каскадных множеств, которые, очевидно, исчерпывают все множества из (1). Следовательно, $\left(\left(Q^{(\text {П) }}\right)^{2} \tilde{L}\right)^{c+h}\left(Q^{(П)}\right)^{2}>0$, что и означает, что $\left.\operatorname{Exp}\left(Q^{(П)}\right)^{2} \tilde{L}\right) \leq c+h+1$.

В случае, когда $f_{i}$ являются блочно равномерными преобразованиями, рассуждения аналогичны. Однако здесь в раунде с номером с вместе с множеством $\bar{W}(1) \bigcup \ldots \bigcup \bar{W}(h)$ будет получено и его замыкание. Значит, в этом случае $\left(\left(Q^{(I)}\right)^{2} \tilde{L}\right)^{c+h-1}\left(Q^{(I)}\right)^{2}>0$. Теорема доказана.

Следствие 5. В условиях и обозначениях из доказательства теоремы 5 имеем:

$$
\begin{aligned}
& c+2 \leq \operatorname{Exp}\left(\left(Q^{(\Pi)}\right)^{2} \tilde{L}\right) \leq c+h+1, \text { если } f_{i} \text { максимально рассеивающие, } u \\
& c+1 \leq \operatorname{Exp}\left(\left(Q^{(\Pi)}\right)^{2} \tilde{L}\right) \leq c+h, \text { если } f_{i} \text { блочно равномерные, }
\end{aligned}
$$

где с-минимальное число, при котором $M_{c}=\{1, \ldots, r\}$ для всех $j=1, \ldots, r$.

Заметим, что указанные в следствии 5 оценки являются, по-видимому, грубыми, так как при их получении до $(c+1)$-го шага мы следили лишь за полнокаскадными множествами максимальных рангов.

Из доказательства теоремы 5 видно, что для минимизации экспоненты матрицы $\left(Q^{(\Pi)}\right)^{2} \tilde{L}$ подстановку $g$ необходимо выбирать так, чтобы параметр $c$ был по возможности наименьшим. Легко видеть, что абсолютный минимум $c$ равен 1 и достигается лишь при $h \leq d$ и для подстановок $g$, которые номера подблоков каждого каскада переводят в номера подблоков всех каскадов. Для этого случая получаем

Следствие 6. Если в $A_{1}$-схеме из теоремы 5 выполняется неравенство $h \leq d$ и подстановка $g$ переводит номера подблоков каждого каскада в номера подблоков всех каскадов, то матрииа $\left(Q^{(\Pi)}\right)^{2} \tilde{L}$ сходится к равномерной матрице, и $\operatorname{Exp}\left(\left(Q^{(\Pi)}\right)^{2} \tilde{L}\right)=4$, если $f_{i}$ максимально рассеивающие, и $\operatorname{Exp}\left(\left(Q^{(П)}\right)^{2} \tilde{L}\right)=3$, если $f_{i}$ блочно равномерные.

Доказательство. Пусть $f_{i}-$ максимально рассеивающие преобразования. В рассматриваемом случае равенство (26) будет выполняться при $c=2$, и на 3-м шаге будет получено замыкание множества $\bar{W}(1) \cup \ldots \cup \bar{W}(h)$. Докажем, что после 4-го шага появятся все множества из (1). 
Пусть, например, $M \subset\{1, \ldots, r\}$ и $M \cap N\left(W_{s}\right) \neq \varnothing$, например, только при $s=1, \ldots, t . \quad$ Выберем $\quad$ множества $R_{i} \in N(\bar{W}(i)), i=1, \ldots, h, \quad$ так, $\quad$ чтобы $g^{-1}\left(R_{1} \cup \ldots \cup R_{h}\right)=N\left(\bar{W}_{1}\right) \cup \ldots \cup N\left(\bar{W}_{t}\right)$. При указанном условии на $g$ такой выбор возможен, причем $R_{i} \neq \varnothing, i=1, \ldots, h$. На 4-м шаге подстановка $g$ переведет множество $V\left(R_{1} \cup \ldots \cup R_{h}\right)$, полученное на предыдущем шаге, в $V\left(N\left(\bar{W}_{1}\right) \cup \ldots \cup N\left(\bar{W}_{t}\right)\right)$, из которого с помощью $f$ получится замыкание $C(\bar{W}(1) \cup \ldots \cup \bar{W}(h))$, которое, очевидно, содержит $V(M)$. Следовательно, $\operatorname{Exp}\left(\left(Q^{(\Pi)}\right)^{2} \tilde{L}\right)=4$.

В случае, когда $f_{i}$ - блочно равномерные преобразования, рассуждения аналогичны, следует лишь учесть, что замыкание множества $\bar{W}(1) \bigcup \ldots \bigcup \bar{W}(h)$ получится на 2-м шаге. Поэтому здесь $\operatorname{Exp}\left(\left(Q^{(\Pi)}\right)^{2} \tilde{L}\right)=3$.

Следствие 7. В $A_{1}$-схеме из теоремы 5 в условиях следствия 6 минимальное число раундов $N$, за которое реализуется 2-транзитивное множество подстановок, равно 9, если $f_{i}$ максимально рассеивающие, $u$ 7, если $f_{i}$ блочно равномерные.

\section{§ 5. О сравнении рассеивающих свойств линейных преобразований}

По результатам предыдущих параграфов для минимального числа $N$ раундов, за которые $\mathrm{A}_{1}$-схема может реализовать 2-транзитивное множество подстановок, имеем следующие соотношения:

$N \leq 5$ - при блочно равномерных рассеивающих преобразованиях;

$N=6$ - при максимально рассеивающих преобразованиях;

$N=7$ - при составных рассеивающих преобразованиях с использованием блочно равномерных преобразований $f_{i}$;

$N=9$ - при составных рассеивающих преобразованиях с использованием максимально рассеивающих преобразований $f_{i}$.

Таким образом, блочно равномерные рассеивающие преобразования обеспечивают наилучшую скорость получения в $\mathrm{A}_{1}$-схемах 2-транзитивного множества подстановок, т. е. минимальную величину экспоненты матрицы $P^{(2)}$ переходных вероятностей пар различных блоков по сравнению с другими рассмотренными преобразованиями. Однако, как было указано во введении, под основным рассеивающим свойством преобразования $L$ в $\mathrm{A}_{1}$ схемах мы понимаем его способность обеспечивать сравнительно быструю сходимость матрицы $P^{(2)}$, или, что то же самое, матрицы $\left(Q^{(П)}\right)^{2} \tilde{L}$, к равномерной матрице, при условии $\left(Q^{(\text {П) }}\right)^{2}>0$. Известно, что малая величина 
экспоненты матрицы способствует и сравнительно быстрой сходимости ее к равномерной матрице. Однако, ниоткуда не следует, что указанное свойство является определяющим для скорости сходимости. Поэтому вопрос о сравнении скоростей сходимости матриц к равномерной матрице требует особого исследования. Вопрос этот с теоретической точки зрения является, как правило, сложным. Экспериментальная проверка в практически интересных случаях также не реальна в силу большого размера блоков. Кроме того, на скорость сходимости матрицы $\left(Q^{(П)}\right)^{2} L$ может влиять и подстановка П. В связи с этим, нам представляется интересным следующий подход, который реально осуществим и позволяет качественно оценивать скорость сходимости матриц при достаточно больших значениях параметров.

Отвлечемся на время от $\mathrm{A}_{1}$-схем и вместо матриц $\left(Q^{(\Pi)}\right)^{2} \tilde{L}$ рассмотрим матрицы вида $\bar{Q} \tilde{L}$, где $\bar{Q}$ - матрица, полученная из $\left(Q^{(\text {()) }}\right)^{2}$ заменой ее диагональных клеток равномерными матрицами тех же размеров. Клетку матрицы $\bar{Q}$, заменившую клетку $\left(Q_{i_{1}, \ldots, i_{s}}\right)^{2}$, обозначим через $\bar{Q}_{i_{1}, \ldots, i_{s}}$. Клетка $\bar{Q}_{i_{1}, \ldots, i_{s}}$ является матрицей размеров $M^{s} \times M^{s}$ с элементами, равными $M^{-s}$, где $M=q^{m}-1$. Отсюда видно, что сама матрица $\bar{Q}$ является сильно неравномерной. Так, например, в ней элементы клетки $\bar{Q}_{1,2, \ldots, m}$ в $M^{m-1}$ раз меньше элементов клеток $\bar{Q}_{i}$.

Будем сравнивать скорости сходимости таких матриц к равномерной матрице при выборе $L$ из некоторых рассмотренных выше классов рассеивающих линейных преобразований путем непосредственного вычисления их элементов . мы 1.

I. Пусть $L-$ блочно равномерное линейное преобразование из теоре-

Матрицы $(\bar{Q} \tilde{L})^{k} \bar{Q}$ при $k=1,2, \ldots$ можно записать в явном виде. Следствие 2 теоремы 1 подсказывает нам, что для более компактной записи этих матриц их целесообразно разбить на $m$ горизонтальных и $m$ вертикальных полос размеров соответственно

$$
C_{m}^{1} M, \quad C_{m}^{2} M^{2}, \ldots, \quad C_{m}^{m} M^{m} .
$$

В итоге матрицы $(\bar{Q} \tilde{L})^{k} \bar{Q}$ разобьются на $m^{2}$ прямоугольных подматриц. Обозначим подматрицы-клетки размеров $C_{m}^{s} M^{s} \times C_{m}^{t} M^{t}$ для матрицы $(\bar{Q} \tilde{L})^{k} \bar{Q}$ в виде $\left(a_{s, t}^{(k)}\right)$. 
Утверждение 4. При введенных обозначениях для любых $s, t \in\{1, \ldots, m\}$ имеют место равенства: 1) $a_{s, t}^{(1)}=\lambda_{s, t} M^{-(s+t)}$; 2) $a_{s, t}^{(k+1)}=\sum_{i=1}^{m} C_{m}^{i} C_{m}^{t} \lambda_{i, t} a_{s, i}^{(k)}$.

Доказательство. Формула 1) следует непосредственно из теоремы 1 , ее следствия 2 и правила умножения матриц. Докажем рекуррентную формулу 2).

Напомним, что умножение матрицы $(\bar{Q} \tilde{L})^{k} \bar{Q}$ на матрицу $\tilde{L}$ равносильно перестановке столбцов матрицы, причем, как следует из теоремы 1 , из $i$-й вертикальной полосы в $t$-ю переставляется $C_{m}^{i} C_{m}^{t} \lambda_{i, t}$ столбцов. А так как каждый элемент, содержащийся в $s$-й горизонтальной и $i$-й вертикальной полосах матрицы $S$, равен $a_{s, i}^{(k)}$, то сумма всех элементов каждой строки из $s$-й горизонтальной полосы, расположенных в $t$-й вертикальной полосе, равна $\sum_{i=1}^{m} C_{m}^{i} C_{m}^{t} \lambda_{i, t} a_{s, i}^{(k)}$. При умножении матрицы $(\bar{Q} \tilde{L})^{k} \bar{Q} \tilde{L}$ на $\bar{Q}$ эта сумма умножается на элемент матрицы $\bar{Q}_{i_{1}, \ldots, i_{t}}$, который равен $M^{-t}$. В итоге получим требуемую формулу для элемента $a_{s, i}^{(k+1)}$. Утверждение 4 доказано.

Для наглядности выпишем общий вид матрицы $S=\bar{Q} \tilde{L} \bar{Q}$ :

$$
S=\left(\begin{array}{ccccc}
\left(\lambda_{1,1} M^{-2}\right) & \left(\lambda_{1,2} M^{-3}\right) & \left(\lambda_{1,3} M^{-4}\right) & \ldots & \left(\lambda_{1, m} M^{-(m+1)}\right) \\
\left(\lambda_{2,1} M^{-3}\right) & \left(\lambda_{2,2} M^{-4}\right) & \left(\lambda_{2,3} M^{-5}\right) & \ldots & \left(\lambda_{2, m} M^{-(m+2)}\right) \\
\left(\lambda_{3,1} M^{-4}\right) & \left(\lambda_{3,2} M^{-5}\right) & \left(\lambda_{3,3} M^{-6}\right) & \ldots & \left(\lambda_{3, m} M^{-(m+3)}\right) \\
\ldots & \ldots \ldots & \ldots & \ldots & \ldots \\
\left(\lambda_{m, 1} M^{-(m+1)}\right) & \left(\lambda_{m, 2} M^{-(m+2)}\right) & \left(\lambda_{m, 3} M^{-(m+3)}\right) & \ldots & \left(\lambda_{m, m} M^{-2 m}\right)
\end{array}\right),
$$

где $\left(\lambda_{i, j} M^{-(i+j)}\right)$ - матрица размера $C_{m}^{i} M^{i} \times C_{m}^{j} M^{j}$ с элементами $\lambda_{i, j} M^{-(i+j)}$.

ПриМЕР 4. Найдем в условиях утверждения 4 матрицы $S=\bar{Q} \tilde{L} \bar{Q}$ и $T=(\bar{Q} \tilde{L})^{3} \bar{Q}$ при $q=2, m=r=3$ и блочно-равномерном рассеивающем преобразовании $L$ из теоремы 1.

Из утверждения 4, пользуясь таблицей 1 для значений $\lambda_{s, t}$ из примера 1, получим:

$$
S=\left(\begin{array}{ccc}
\left(7^{-2}\right) & \left(7^{-3}\right) & \left(7^{-4}\right) \\
\left(7^{-3}\right) & \left(7.7^{-4}\right) & \left(25.7^{-5}\right) \\
\left(7^{-4}\right) & \left(25.7^{-5}\right) & \left(265.7^{-6}\right)
\end{array}\right)
$$

числа строк 1-й, 2-й и 3 -й полосы матрицы $S$ равны: $3 \cdot 7,3 \cdot 7^{2}, 7^{3}$ соответственно. 
Для нахождения матрицы $T$ выпишем сначала формулу для $a_{s, t}^{(3)}$ в общем случае (пользуясь утверждением 4):

$$
a_{s, t}^{(3)}=M^{-(s+t)} \sum_{j=1}^{m} C_{m}^{j} \lambda_{j, t} M^{-j} \sum_{i=1}^{m} C_{m}^{i} \lambda_{i, j} \lambda_{s, i} M^{-i}
$$

Теперь, произведя необходимые промежуточные вычисления с точностью до 0,001, получим:

$$
T \approx 7^{-4}\left(\begin{array}{ccc}
(13,53) & (5,66) & (3,75) \\
(5,66) & (4,87) & (4,57) \\
(3,75) & (4,57) & (4,81)
\end{array}\right)
$$

II. Пусть $L-$ максимально рассеивающее преобразование.

Здесь подматрицы-клетки размера $C_{m}^{s} M^{s} \times C_{m}^{t} M^{t}$ для матрицы $(\bar{Q} \tilde{L})^{k} \bar{Q}$ обозначим через $\left(b_{s, t}^{(k)}\right)$ и выпишем для них рекуррентные формулы.

Утверждение 5. При введенных обозначениях для любых $s, t \in\{1, \ldots, m\}$ имеют место равенства: 1) $b_{s, t}^{(1)}=\mu_{s, t} M^{-(s+t)}$; 2) $b_{s, t}^{(k+1)}=\sum_{i=1}^{m} C_{m}^{i} C_{m}^{t} \mu_{i, t} a_{s, i}^{(k)}$.

Доказательство утверждения 5 получается из доказательства утверждения 4 заменой чисел $\lambda_{i, j}$ на $\mu_{i, j}$ и числа $m$ на $r$.

В частности, здесь матрица $S_{1}=\bar{Q} \tilde{L} \bar{Q}$, с учетом равенств $\mu_{i, j}=0$ при $j \leq r-i$, выглядит следующим образом:

$$
S_{1}=\left(\begin{array}{ccccc}
(0) & (0) & \ldots & (0) & \left(\mu_{1, r} M^{-(r+1)}\right) \\
(0) & (0) & \ldots & \left(\mu_{2, r-1} M^{-(r+1)}\right) & \left(\mu_{2, r} M^{-(r+2)}\right) \\
\ldots & \ldots & \ldots & \ldots & \ldots \\
(0) & \left(\mu_{r-1,2} M^{-(r+1)}\right) & \ldots & \left(\mu_{r-1, r-1} M^{-(2 r-2)}\right) & \left(\mu_{r-1, r} M^{-(2 r-1)}\right) \\
\left(\mu_{r, 1} M^{(r+1)}\right) & \left(\mu_{r, 2} M^{-(r+2)}\right) & \ldots & \left(\mu_{r, r-1} M^{-(2 r-1)}\right) & \left(\mu_{r, r} M^{-2 r}\right)
\end{array}\right) .
$$

ПримЕР 5. Найдем матрицы $S_{1} u T_{1}=(\bar{Q} \tilde{L})^{3} \bar{Q}$ при $q=2, m=r=3$ и максимально рассеивающем преобразовании $L=L_{A}$ из примера 3.

Здесь матрицы $T_{1}$ и $S_{1}$ при таком же разбиении на полосы и такой же точности вычислений, что и в примере 4 , имеют вид:

$$
S_{1}=\left(\begin{array}{ccc}
(0) & (0) & \left(7^{-3}\right) \\
(0) & \left(7^{-3}\right) & \left(4 \cdot 7^{-4}\right) \\
\left(7^{-3}\right) & \left(4 \cdot 7^{-4}\right) & \left(34 \cdot 7^{-5}\right)
\end{array}\right), T_{1} \approx 7^{-4}\left(\begin{array}{ccc}
(4,86) & (4,49) & (4,78) \\
(4,49) & (4,83) & (4,65) \\
(4,8) & (4,7) & (4,71)
\end{array}\right)
$$


ЗАМЕЧАНИЕ 2. Из примеров 4, 5 видно, что максимально рассеивающая матрица обеспечивает более быструю сходимость матрицы $\bar{Q} \tilde{L}$ к равномерной матрице по сравнению с блочно равномерной матрицей. И этот факт имеет простое объяснение. Блочно равномерное преобразование более равномерно рассеивает элементы множеств (1) по всем множествам из (1) по сравнению с максимально рассеивающим преобразованием, однако при этом слабо учитываются порядки множеств (1). Так, например, преобразование из теоремы 1 отображает из множеств $V(i)$ в множество $V\left(j_{1}, \ldots, j_{t}\right)$ ровно $(q-1)^{t}$ элементов, и значит, при $q=2$ - по одному элементу в каждое из множеств (1), хотя мощности множества $V\left(j_{1}, \ldots, j_{t}\right)$ в данном случае при $t=1$ и $t=r$ равны соответственно $2^{m}-1$ и $\left(2^{m}-1\right)^{r}$. Поэтому в матрице $S$ при блочно-равномерном преобразовании самые большие элементы находятся в левом верхнем углу, они и создают неравномерность в степенях матрицы. В то же время максимально рассеивающее преобразование отображает все элементы из $V(i)$ в одно и то же, самое мощное, множество $V(1, \ldots, r)$, что дает возможность более равномерного рассеивания элементов на следующем раунде.

III. Пусть $L-$ составное рассеивающее преобразование.

Здесь, как и выше, для нахождения матрицы $\bar{Q} \tilde{L}$ необходимо знать числа (6). В общем случае вся информация о числах (6) размещается в матрице размера $\left(2^{r}-1\right) \times\left(2^{r}-1\right)$. Однако в п. I, II всю эту информацию оказалось возможным разместить в матрицах размеров $r \times r$, поскольку число (6) однозначно определялось значениями $s, t$. При использовании составного рассеивающего преобразования в общем случае аналогичный факт не имеет места, и потому матрицей размеров $r \times r$ здесь не обойтись. Кроме того, здесь матрица $\bar{Q} \tilde{L}$ зависит и от разбиения блоков на каскады, и от выбора подстановки $g$. В связи с этим в данном случае сравнение скорости сходимости матрицы $\bar{Q} \tilde{L}$ к равномерной со случаями I, II затруднительно. Такое сравнение скоростей возможно при конкретных подстановках $g$, что в этой работе не рассматривается.

ЗАМЕЧАНИЕ 3. Заметим, что при надлежащем выборе подстановок $\Pi_{i}$ матрицы $Q_{i}^{2}$ могут быть достаточно близкими к равномерным, так, что в них максимальный элемент лишь в 2-3 раза отличается от минимального. Отсюда, учитывая указанную выше огромную разницу между элементами разных клеток матрицы $\left(Q^{(\Pi)}\right)^{2}$, сохраняющуюся при переходе к $\bar{Q}$, можно предполагать, что имеющиеся в матрице $\left(Q^{(\text {П) }}\right)^{2}$ отклонения от равномерности не будут существенно влиять на различие в скоростях сходимости мат- 
риц $\left(Q^{(П)}\right)^{2} \tilde{L}$ и $\bar{Q} \tilde{L}$ к равномерной матрице. В связи с этим скорость сходимости матрицы $\bar{Q} \tilde{L}$ можно рассматривать как качественную оценку скорости сходимости матрицы $\left(Q^{(П)}\right)^{2} \tilde{L}$.

Указанное предположение подтверждают, в частности, компьютерные вычисления матрицы $\left(\left(Q^{(\Pi)}\right)^{2} \tilde{L}\right)^{3} Q^{(\text {П) }}$ для $\mathrm{A}_{1}$-схем из примеров 4,5 при выборе в качестве подстановок $\Pi_{i}$ подстановки обращения ненулевых элементов поля $G F\left(2^{3}\right)$. Для наглядности выпишем матрицы $T^{\prime}$ и $T_{1}^{\prime}$ минимальных элементов клеток матриц $\left(\left(Q^{(\Pi)}\right)^{2} \tilde{L}\right)^{3} Q^{(\Pi)}$, соответствующих клеткам матриц $T$ и $T_{1}$ примеров 4,5 :

$$
T^{\prime}=\left(\begin{array}{ccc}
14,55 & 5,02 & 2,82 \\
5,02 & 4,74 & 4,16 \\
2,82 & 4,16 & 4,76
\end{array}\right), \quad T_{1}^{\prime}=\left(\begin{array}{ccc}
4,85 & 4,44 & 4,77 \\
4,45 & 4,81 & 4,64 \\
4,76 & 4,64 & 4,71
\end{array}\right) .
$$

ЗАМЕЧАНИЕ 4. Выполнение условия $\left(Q_{i}\right)^{2}>0$, использованного нами при изучении $\mathrm{A}_{1}$-схем, достигалось за счет выбора подходящих подстановок $\Pi_{i}$ и за счет такого режима работы схемы, когда нетривиальные линейные преобразования применяются лишь в четных раундах. Однако такой режим работы может повлечь необходимость увеличения числа раундов для обеспечения стойкости схемы по отношению к некоторым методам анализа. В связи с этим может оказаться полезным другой путь, заключающийся в замене матриц $Q_{i}$ на положительные матрицы за счет незначительного изменения Асхемы. Из [1] следует, что этого нельзя добиться за счет лучшего выбора подстановок $\prod_{i}$. Однако этого можно добиться путем замены группы $\Sigma_{m}$ какимлибо другим, более мощным, множеством преобразований. Например, вместо группы $\Sigma_{m}$ можно использовать объединение нескольких смежных классов полной аффинной группы $\operatorname{GL}(m, q)$ по подгруппе $\Sigma_{m}$, выбор которых из заданного множества производится в каждом раунде по независимому ключу. Введение такой динамики в A-схему усложняет её с точки зрения XSL-метода анализа блочных шифров.

В заключение выражаю признательность рецензентам А. М. Зубкову, А. С. Кузьмину, Ф. М. Малышеву, Б. А. Погорелову и А. Е. Тришину за полезные замечания к первоначальному варианту данной статьи.

\section{Список литературы}

1. Глухов М. М. О 2-транзитивных произведениях регулярных групп подстановок. - Труды по дискретной математике, т. 3. М.: 2000. С. 37-52.

2. Глухов М. М., Елизаров В. П., Нечаев А. А. Алгебра, т. ІІ. - М.: «ГелиосAPB», 2003. 
3. Мальшев Ф. М. Дважды транзитивные XSL-семейства подстановок. Матем. вопросы криптографии, т. 1, вып. 2, с. 93-106.

4. Мак-Вильямс Ф. Д., Слоэн Н. Д. А. Теория кодов, исправляющих ошибки. - М.: Связь, 1979.

5. Сачков В. Н. Вероятностные методы в комбинаторном анализе. - М.: Наука, 1978.

6. Daemen J., Rijmen V. The Rijndael block cipher. - AES Proposal, 1998. P. 1-37.

7. Wielandt H. Finite permutation groups. - N.Y.-London: Acad. Press, 1984. 
